This article is published with open access at Springerlink.com

\title{
Densification of FL chains via residuated frames
}

\author{
Paolo Baldi and Kazushige Terui
}

\begin{abstract}
We introduce a systematic method for densification, i.e., embedding a given chain into a dense one preserving certain identities, in the framework of FL algebras (pointed residuated lattices). Our method, based on residuated frames, offers a uniform proof for many of the known densification and standard completeness results in the literature. We propose a syntactic criterion for densification, called semianchoredness. We then prove that the semilinear varieties of integral FL algebras defined by semi-anchored equations admit densification, so that the corresponding fuzzy logics are standard complete. Our method also applies to (possibly non-integral) commutative FL chains. We prove that the semilinear varieties of commutative FL algebras defined by knotted axioms $x^{m} \leq x^{n}$ (with $m, n>1$ ) admit densification. This provides a purely algebraic proof to the standard completeness of uninorm logic as well as its extensions by knotted axioms.
\end{abstract}

\section{Introduction}

Given a class $\mathrm{K}$ of ordered algebras and a chain $\mathbf{A}$ (i.e., a totally ordered algebra) in it, we would like to embed $\mathbf{A}$ into a dense chain in the same class $\mathrm{K}$. This construction, referred to as densification, is important both on its own and as a key step towards standard completeness of various fuzzy logics, i.e., a completeness theorem with respect to the valuations of propositional variables into the real unit interval $[0,1]$. Many fuzzy logics fall into the class of substructural logics, whose algebraic semantics are given by $F L$ algebras (or pointed residuated lattices) [19]. We thus consider densification of FL chains and standard completeness of associated fuzzy logics. Some classes of FL chains, such as Gödel chains, are well known to be densifiable, while others, such as Boolean algebras, are clearly not densifiable; see, e.g., [21] for background. However, there is no general criteria for classifying densifiable and non-densifiable FL chains.

Our aim in this paper is not to prove a new result, but rather to provide a uniform, algebraic account of densification. This is important since standard completeness is often proved by a proof theoretic argument, known as density elimination in the hypersequent calculus $[1,26,11,12,4]$. While density

Presented by C. Tsinakis.

Received June 20, 2014; accepted in final form January 12, 2015.

2010 Mathematics Subject Classification: Primary: 03B47; Secondary: 06F05, 03G10, $08 \mathrm{~B} 15$.

Key words and phrases: substructural logic, fuzzy logic, Gentzen systems, residuated frames, residuated lattices, standard completeness.

Partly supported by the FWF project START Y544-N23, the project GAP202/10/1826 and JSPS KAKENHI 25330013. 
elimination is no doubt an interesting application of proof theory, it obscures the algebraic essence of densification. Even when densification is proved algebraically (as in $[23,24,21]$ ), it is not clear to what extent the employed technique generalizes. To fulfill our goal, we employ residuated frames [18], that are effective devices to construct (complete) FL algebras with various properties. They are also a key to connect proof theory with algebraic studies; for instance, one can naturally define a residuated frame $\mathbf{W}$ from the sequent calculus $\mathbf{F L}$, so that validity in the dual algebra $\mathbf{W}^{+}$directly implies cut-free derivability in FL. This strong connection allowed us to prove that for a certain class of substructural logics, a strong form of cut-admissibility (proof theory) is equivalent to closure under completions (algebra), thus promoting a new approach to substructural logics, dubbed algebraic proof theory for substructural logics $[9,10]$.

In this paper, we use residuated frames to densify a given FL chain, preserving certain identities. Although our argument was originally inspired by density elimination following the spirit of algebraic proof theory, the resulting construction can be understood without any reference to proof theory: it provides a purely algebraic account. Further steps in a purely algebraic analysis of densification can be found in [17].

The rest of this paper is organized as follows. Section 2 discusses densification in a general setting, and Section 3 specializes it to FL algebras. Section 4 reviews residuated frames, and Section 5 applies them to densify integral FL chains. This encompasses the standard completeness of monoidal t-norm logic and its noncommutative variant $[23,24]$. Section 6 then addresses a more involved case: (possibly non-integral) commutative FL chains. It provides a purely algebraic proof of the standard completeness of uninorm logic, for which only proof theoretic arguments were previously known [26].

Section 7 recalls the concept of a substructural hierarchy $[7,8,9,10]$, that is useful to classify equations in the language of FL. Based on the hierarchical classification, we introduce the class of semi-anchored $\mathcal{P}_{3}$ equations in Section 8 , and prove a general result that every nontrivial semilinear variety of integral FL algebras defined by semi-anchored $\mathcal{P}_{3}$ equations admits densification and standard completeness. We then turn our attention to varieties of commutative FL algebras in Section 9. We prove that every semilinear variety of commutative FL algebras defined by knotted axioms $x^{m} \leq x^{n}$ with $m, n>1$ admits densification and standard completeness. These results are

again inspired by the proof theoretic arguments in $[4,2]$. We conclude the paper with some remarks and open problems.

\section{Densifiability}

We begin with a general consideration of densification and standard completeness. In the sequel, we assume that every algebra $\mathbf{A}$ comes equipped with 
an order $\leq_{\mathbf{A}}$ defined by equations (e.g., $x \leq_{\mathbf{A}} y \Leftrightarrow x=x \wedge y$ if $\mathbf{A}$ has a lattice reduct).

Definition 2.1. Let $\mathbf{A}$ be a chain, i.e., $\leq_{\mathbf{A}}$ is a total order, of cardinality $\kappa>1$; $\mathbf{A}$ is dense if $g<h(g, h \in A)$ implies $g<p<h$ for some $p \in A$. Otherwise A contains a gap, that is, a pair of elements $g, h \in A$ such that $g<h$ and there is no element $p \in A$ with $g<p<h$.

A chain $\mathbf{B}$ fills a gap $(g, h)$ of $\mathbf{A}$ if there is an embedding $e: \mathbf{A} \longrightarrow \mathbf{B}$ and an element $p \in B$ such that $e(g)<p<e(h)$. A nontrivial variety $\mathrm{V}$ is said to be densifiable if every gap of a chain in $\mathrm{V}$ can be filled by another chain in $\mathrm{V}$.

Notice that by filling a gap, one may introduce some undesirable elements that have nothing to do with the gap. Nevertheless, densifiability is a sufficient condition for densification.

Proposition 2.2. Let $L$ be a language of algebras and $\mathrm{V}$ a densifiable variety of type L. Then every chain $\mathbf{A}$ of cardinality $\kappa>1$ in $\mathrm{V}$ is embeddable into a dense chain of cardinality $\kappa+\aleph_{0}+|L|$ in $\mathrm{V}$.

Proof. For simplicity, let us assume $\kappa,|L| \leq \aleph_{0}$; the argument below clearly works also for an arbitrary $\kappa>1$ and $L$, provided that one uses the axiom of choice. Let $X$ be a countable set of variables and $T=T(X)$ the set of terms in the language $L$ over $X$. Let $\left(t_{0}, u_{0}\right),\left(t_{1}, u_{1}\right), \ldots$ be a countable sequence of elements of $T^{2}$ such that each $(t, u) \in T^{2}$ occurs infinitely many times in it.

For each $n \in \mathbb{N}$, we define a chain $\mathbf{B}_{n}$ in $\mathrm{V}$ as well as a partial function $f_{n}: X \rightarrow B_{n}$. Let $\mathbf{B}_{0}:=\mathbf{A}$ and $f_{0}$ be any surjective partial function onto $A$ such that $X \backslash \operatorname{dom}\left(f_{0}\right)$ is infinite.

For $n \geq 0$, if one of $f_{n}\left(t_{n}\right), f_{n}\left(u_{n}\right)$ is undefined or $f_{n}\left(t_{n}\right) \nless f_{n}\left(u_{n}\right)$, then let $\mathbf{B}_{n+1}:=\mathbf{B}_{n}$ and $f_{n+1}:=f_{n}$.

Otherwise, let $x$ be a variable taken from $X \backslash \operatorname{dom}\left(f_{n}\right)$. If there is $p \in B_{n}$ such that $f_{n}\left(t_{n}\right)<p<f_{n}\left(u_{n}\right)$, then let $\mathbf{B}_{n+1}:=\mathbf{B}_{n}$. If not, let $\mathbf{B}_{n+1}$ be a chain in $\mathrm{V}$ that fills the gap $\left(f_{n}\left(t_{n}\right), f_{n}\left(u_{n}\right)\right)$ by $p \in B_{n+1}$. We assume $B_{n} \subseteq B_{n+1}$ and define $f_{n+1}: X \rightarrow B_{n+1}$ by extending $f_{n}$ with $f_{n+1}(x):=p$.

Let $\mathbf{B}:=\bigcup \mathbf{B}_{n}, f:=\bigcup f_{n}$, and $\mathbf{C}$ be the subalgebra of $\mathbf{B}$ generated by $f[X]$ (so that $C=f[T]$ ). Clearly, $\mathbf{C}$ is a countable chain in $\mathbf{V}$ that has $\mathbf{A}$ as subalgebra since $A \subseteq f[X]$. Moreover, $\mathbf{C}$ is dense since for every pair $(g, h) \in C^{2}$ with $g<h$, there is $n \in \mathbb{N}$ such that $g=f_{n}\left(t_{n}\right)$ and $h=f_{n}\left(u_{n}\right)$, so that we have $g<f_{n+1}(x)<h$.

Given a class $\mathrm{K}$ of algebras of the same type, the semantic consequence relation $\models_{\mathrm{K}}$ is defined as usual. Namely, given a set $E \cup\{s=t\}$ of equations in the language of $\mathrm{K}, E \models_{\mathrm{K}} s=t$ holds if $E$ entails $s=t$ in every algebra $\mathbf{A} \in \mathrm{K}$.

A variety $\mathrm{V}$ is semilinear if $\models \mathrm{v}=\models \mathrm{V}_{C}$, where $\mathrm{V}_{C}$ consists of all chains in $\mathrm{V}$. This is equivalent to saying that every subdirectly irreducible algebra in $\mathrm{V}$ is a chain (cf. [21]). We say $\mathrm{V}$ is (strongly) standard complete if $\models \mathrm{V}=\models \mathrm{V}_{[0,1]}$, 
where $\mathrm{V}_{[0,1]}$ consists of all standard chains in $\mathrm{V}$, namely those over the real unit interval $([0,1], \leq)$. This conforms to the terminology in fuzzy logics under the identification of an (algebraizable) logic with the corresponding variety. There are actually two weaker notion of standard completeness. The finite strong standard completeness defined as $E \models \mathrm{v} s=t$ iff $E \models \mathrm{v}_{[0,1]}$ for $E$ a finite set of equations and the weak standard completeness defined as $\emptyset \models \mathrm{v} s=t$ iff $\emptyset \models \mathrm{v}_{[0,1]} s=t$. In the rest of this paper, we mean by standard completeness the strongest version.

We need a few concepts concerning completions. Given an algebra A, a completion of $\mathbf{A}$ consists of a complete algebra $\mathbf{B}$ together with an embedding $e: \mathbf{A} \longrightarrow \mathbf{B}$. A completion $(\mathbf{B}, e)$ is join-dense if $x=\bigvee\left\{a \in e[A]: a \leq_{\mathbf{B}} x\right\}$ and meet-dense if $x=\bigwedge\left\{a \in e[A]: x \leq_{\mathbf{B}} a\right\}$, for every $x \in B$. A join-dense and meet-dense completion is called a MacNeille completion. It is known that the lattice reduct of a MacNeille completion is uniquely determined (up to isomorphism that fixes $A$ ) by join and meet density $[5,29]$. For instance, the MacNeille completion of the rational unit interval $\left([0,1]_{\mathbb{Q}}, \leq\right)$ is just $([0,1], \leq)$.

Proposition 2.3. Let $L$ be a finite or countable language of algebras and $\mathrm{V}$ a variety of type $L$. If $\mathrm{V}$ is semilinear, densifiable and every chain in $\mathrm{V}$ has a MacNeille completion in it, then $\mathrm{V}$ is standard complete.

Proof. Let $E \cup\{s=t\}$ be a set of equations, and suppose that $E \not \neq v s=t$. By semilinearity, there is a finite or countable chain $\mathbf{A}$ such that $E \|_{\mathbf{A}} s=t$. By Proposition 2.2, $\mathbf{A}$ is embeddable into a countable dense chain $\mathbf{B}$ in $\mathrm{V}$. It is well known that $\left(B, \leq_{\mathbf{B}}\right)$ is isomorphic to one of $(0,1)_{\mathbb{Q}},(0,1]_{\mathbb{Q}},[0,1)_{\mathbb{Q}}$, and $[0,1]_{\mathbb{Q}}$, whose MacNeille completion is $[0,1]$. Hence, $\mathbf{B}$ is embeddable into a standard chain $\mathbf{C}$ in $\mathrm{V}_{[0,1]}$, and we have $E \not \neq \mathrm{V}_{[0,1]} s=t$.

Hence, there are two key factors for standard completeness: densifiability and closure under MacNeille completions.

\section{FL algebras}

In this section, we recall the concept of an FL algebra. A standard reference on this topic is [19].

Definition 3.1. A residuated lattice is an algebra $\mathbf{A}=(A, \wedge, \vee, \cdot, \backslash, /, 1)$ such that $(A, \wedge, \vee)$ is a lattice, $(A, \cdot, 1)$ is a monoid, and for all $x, y, z \in A$,

$$
x \cdot y \leq z \Longleftrightarrow y \leq x \backslash z \Longleftrightarrow x \leq z / y \text {. }
$$

An $F L$ algebra is a residuated lattice $\mathbf{A}$ with a distinguished element $0 \in A$. The constant 0 is used to define negations: $\sim x:=x \backslash 0,-x:=0 / x$. $\mathbf{A}$ is

(e) commutative if $x \cdot y=y \cdot x$ for all $x, y \in A$,

(c) contractive if $x \leq x \cdot x$ for every $x \in A$,

(i) integral if 1 is the greatest element,

(o) 0-bounded if 0 is the least element. 
We remark that $\mathbf{A}$ is a chain if and only if the following communication property holds for all $x, y, z, w \in A$ :

$$
x \leq z \text { and } y \leq w \Longrightarrow x \leq w \text { or } y \leq z .
$$

Also important is the fact that if $(g, h)$ is a gap in a FL chain, then we have $h \backslash g<1$. Indeed, $1 \leq h \backslash g$ would imply $h \leq g$.

The two divisions $\backslash$ and / coincide in any commutative FL algebra. So we write $x \rightarrow y:=x \backslash y=y / x$ in that case.

We write FL for the variety of FL algebras and use subscripts e, c, i, o to indicate the properties $(e),(c),(i),(o)$ above. For instance, $\mathrm{FL}_{\mathrm{ei}}$ denotes the variety of commutative integral FL algebras. It is known (cf. [19, 21]) that a variety of FL algebras is semilinear if and only if it satisfies the four-variable equation $\lambda_{a}(x \vee y \backslash y) \vee \rho_{b}(x \vee y \backslash x)=1$, where $\lambda_{a}$ and $\rho_{b}$ are conjugate operators defined by: $\lambda_{a}(x):=(a \backslash x a) \wedge 1$ and $\rho_{b}(x):=(b x / b) \wedge 1$.

Given a variety $\mathrm{V}$ of FL algebras, we denote by $\mathrm{V}^{\ell}$ the subvariety obtained by imposing the above equation. Notice that it is equivalent to the familiar prelinearity axiom $(x \rightarrow y) \vee(y \rightarrow x)=1$ in $\mathrm{FL}_{\mathrm{ei}}$ algebras.

Unfortunately, $\mathrm{FL}^{\ell}$ is not standard complete (cf. [31, 21]). On the other hand, it is not hard to see that every subvariety of $\mathrm{FL}_{\mathrm{i}}^{\ell}$ defined by a combination of $(e),(c),(o)$ is densifiable and closed under MacNeille completions, so is standard complete [23, 24].

A short proof of the densifiability of $\mathrm{FL}_{\mathrm{i}}^{\ell}$ is as follows. Let $\mathbf{A}$ be an integral FL chain with a gap $(g, h)$. We insert a new element $p$ between $g$ and $h$ : take $A^{p}:=A \cup\{p\}$, with $g<p<h$. The meet and join operations are naturally extended to $A^{p}$. To extend multiplication - and divisions $\backslash, /$, note that for every $a \in A$, either $a h=h$ or $a h \leq g$ holds. For every $a \in A$, we define:

$$
\begin{aligned}
& p \cdot p:=p \text { if } h^{2}=h, \text { and } p \cdot p:=h^{2}, \text { if } h^{2} \leq g ; \\
& a \cdot p:=p \text { if } a h=h, \text { and } a \cdot p:=a h, \text { if } a h \leq g ; \\
& p \backslash p:=1, \text { and } p \backslash a:=h \backslash a ; \\
& a \backslash p:=p \text { if } a h=h, \text { and } a \backslash p:=a \backslash g, \text { if } a h \leq g .
\end{aligned}
$$

The other cases $p \cdot a, p / p, a / p$, and $p / a$ are defined analogously.

This gives rises to a new algebra $\mathbf{A}^{p}$ in $\mathrm{FL}_{i}^{\ell}$ that fills the gap $(g, h)$ of $\mathbf{A}$. While it is possible to check the correctness manually, our approach is rather to derive $\mathbf{A}^{p}$ by a general residuated frame construction (Section 5). Our approach will explain the rationale behind $\mathbf{A}^{p}$, and provide a generic recipe for proving further standard completeness results. It also leads to an algebraic proof of the standard completeness of uninorm logic [26] in Section 6.

\section{Residuated frames and MacNeille completions}

Just as Kripke frames are useful devices to build various Heyting and modal algebras, residuated frames are useful devices to build various FL algebras. In 
this section, we introduce residuated frames and recall some relevant facts from $[18,9]$.

Definition 4.1. A frame $\mathbf{W}$ (for $\mathrm{FL}$ algebras) is a tuple $\left(W, W^{\prime}, N, \circ, \varepsilon, \epsilon\right)$ where $(W, \circ, \varepsilon)$ is a monoid, $N \subseteq W \times W^{\prime}$, and $\epsilon \in W^{\prime}$. It is residuated if there are functions $\|: W \times W^{\prime} \longrightarrow W^{\prime}$ and $/ / W^{\prime} \times W \longrightarrow W^{\prime}$ such that

$$
x \circ y N z \Longleftrightarrow y N x \| z \Longleftrightarrow x N z / / y \text {. }
$$

We often omit $\circ$ and write $x y$ for $x \circ y$.

Given a frame $\mathbf{W}=\left(W, W^{\prime}, N, \circ, \varepsilon, \epsilon\right)$, there is a canonical way to make it residuated: let $\tilde{W}^{\prime}:=W \times W^{\prime} \times W$ and define $\tilde{N} \subseteq W \times \tilde{W}^{\prime}$ by

$$
x \tilde{N}\left(v_{1}, z, v_{2}\right) \Longleftrightarrow v_{1} x v_{2} N z \text {. }
$$

Then $\tilde{\mathbf{W}}:=\left(W, \tilde{W}^{\prime}, \tilde{N}, \circ, \varepsilon,(\varepsilon, \epsilon, \varepsilon)\right)$ is a residuated frame, since

$$
\begin{aligned}
x \circ y \tilde{N}\left(v_{1}, z, v_{2}\right) & \Longleftrightarrow v_{1} x y v_{2} N z \\
& \Longleftrightarrow y \tilde{N}\left(v_{1} x, z, v_{2}\right) \Longleftrightarrow x \tilde{N}\left(v_{1}, z, y v_{2}\right) .
\end{aligned}
$$

As we have said at the beginning, the primary purpose of residuated frames is to build residuated lattices. We now describe the construction. Let $\mathbf{W}=$ $\left(W, W^{\prime}, N, \circ, \varepsilon, \epsilon\right)$ be a residuated frame. Given $X, Y \subseteq W$ and $Z \subseteq W^{\prime}$, let

$$
\begin{aligned}
X \circ Y & :=\{x \circ y: x \in X, y \in Y\}, \\
X^{\triangleright} & :=\left\{z \in W^{\prime}: X N z\right\}, \\
Z^{\triangleleft} & :=\{x \in W: x N Z\},
\end{aligned}
$$

where $X N z$ iff $x N z$ for every $x \in X$, and $x N Z$ iff $x N z$ for every $z \in Z$.

We write $x^{\triangleright}$ and $z^{\triangleleft}$ instead of $\{x\}^{\triangleright}$ and $\{z\}^{\triangleleft}$. The pair $(\triangleright, \triangleleft)$ forms a Galois connection: $X \subseteq Z^{\triangleleft} \Longleftrightarrow X^{\triangleright} \supseteq Z$, so that $\gamma(X):=X^{\triangleright \triangleleft}$ defines a closure operator on $\mathcal{P}(W)$ (the powerset of $W$ ):

(1) $X \subseteq \gamma(X)$,

(2) $X \subseteq Y \Longrightarrow \gamma(X) \subseteq \gamma(Y)$,

(3) $\gamma(\gamma(X))=\gamma(X)$.

Furthermore, $\gamma$ is a nucleus, namely it satisfies

(4) $\gamma(X) \circ \gamma(Y) \subseteq \gamma(X \circ Y)$.

It is for this property that a frame has to be residuated.

Let $\mathcal{P}(W)$ be the powerset of $W$ and $\gamma[\mathcal{P}(W)] \subseteq \mathcal{P}(W)$ be its image under $\gamma$. Then a set $X$ belongs to $\gamma[\mathcal{P}(W)]$ iff it is Galois-closed, namely $X=\gamma(X)$ iff $X=Z^{\triangleleft}$ for some $Z \subseteq W^{\prime}$. For $X, Y \in \mathcal{P}(W)$, let

$$
\begin{aligned}
X \circ_{\gamma} Y & :=\gamma(X \circ Y), & X \cup_{\gamma} Y:=\gamma(X \cup Y), \\
X \backslash Y & :=\{y: X \circ\{y\} \subseteq Y\}, & Y / X:=\{y:\{y\} \circ X \subseteq Y\} .
\end{aligned}
$$

Proposition 4.2 ([18]). Let $\mathbf{W}=\left(W, W^{\prime}, N, \circ, \varepsilon, \epsilon\right)$ be a residuated frame. Then the dual algebra defined by $\mathbf{W}^{+}:=\left(\gamma[\mathcal{P}(W)], \cap, \cup_{\gamma}, \circ_{\gamma}, \backslash, /, \gamma(\{\varepsilon\}), \epsilon^{\triangleleft}\right)$ is a complete FL algebra. 
As an example, let $\mathbf{A}=(A, \wedge, \vee, \cdot, \backslash, /, 1,0)$ be an FL algebra. Then we may define a frame by $\mathbf{W}_{\mathbf{A}}:=(A, A, N, \cdot 1,0)$, where $N$ is the lattice ordering $\leq$ of $\mathbf{A} . \mathbf{W}_{\mathbf{A}}$ is residuated precisely because $\mathbf{A}$ is residuated:

$$
a \cdot b N c \Longleftrightarrow b N a \backslash c \Longleftrightarrow a N c / b \text {. }
$$

Hence, by the previous proposition, $\mathbf{W}_{\mathbf{A}}^{+}$is a complete $\mathrm{FL}$ algebra. We want $\mathbf{W}_{\mathbf{A}}^{+}$to be commutative (resp. contractive, integral, 0-bounded, totally ordered) whenever $\mathbf{A}$ is. The following rules ensure that.

$$
\begin{array}{cc}
\frac{x y z}{y x N z}\left(e^{N}\right) & \frac{x x N z}{x N z}\left(c^{N}\right) \quad \frac{\varepsilon N z}{x N z}\left(i^{N}\right) \\
\frac{x N \epsilon}{x N z}\left(o^{N}\right) & \frac{x N z \text { and } y N w}{x N w \text { or } y N z}\left(\operatorname{com}^{N}\right)
\end{array}
$$

It is clear that $\mathbf{W}_{\mathbf{A}}$ satisfies $\left(e^{N}\right)\left(\operatorname{resp} .\left(c^{N}\right),\left(i^{N}\right),\left(o^{N}\right),\left(\operatorname{com}^{N}\right)\right)$, whenever A is commutative (resp. contractive, integral, 0-bounded, totally ordered). These properties are in turn propagated to the dual algebra $\mathbf{W}_{\mathbf{A}}^{+}$. This holds for any residuated frame.

Lemma 4.3. Let $\mathbf{W}$ be a residuated frame. If $\mathbf{W}$ satisfies $\left(e^{N}\right)$ (resp. $\left(c^{N}\right)$, $\left.\left(i^{N}\right),\left(o^{N}\right),\left(c^{N} m^{N}\right)\right)$, then $\mathbf{W}^{+}$is commutative (resp. contractive, integral, 0-bounded, totally ordered).

Proof. Let us only prove that $\left(\mathrm{com}^{N}\right)$ implies $\mathbf{W}^{+}$being totally ordered. Suppose that there are $X, Y \in \gamma[\mathcal{P}(W)]$ for which $X \nsubseteq Y$ and $Y \nsubseteq X$. The former means that there are $x \in X$ and $w \in Y^{\triangleright}$ such that $x N w$ does not hold (since $Y=Y^{\triangleright \triangleleft}$ ). Similarly, the latter means that there are $y \in Y$ and $z \in X^{\triangleright}$ such that $y N z$ does not hold. On the other hand, we have $x N z$ and $y N w$ by definition of $X^{\triangleright}, Y^{\triangleright}$. Hence, the rule $\left(\mathrm{com}^{N}\right)$ implies that at least one of $x N w$ and $y N z$ should hold, a contradiction.

Note also that $\mathbf{W}^{+}$is complete, hence it is always a bounded FL algebra. Finally, we would like to have an embedding of $\mathbf{A}$ into $\mathbf{W}_{\mathbf{A}}^{+}$. More generally, let A be an FL algebra and $\mathbf{W}=\left(W, W^{\prime}, N, \circ, \varepsilon, \epsilon\right)$ a residuated frame. Suppose that there are injections $i: A \longrightarrow W$ and $i^{\prime}: A \longrightarrow W^{\prime}$ by means of which we identify $A$ with a subset of $W$ and of $W^{\prime}$. In such a situation, the rules in Figure 1, called Gentzen rules, ensure the existence of a homomorphism.

Lemma 4.4 ([18]).

(1) If $(\mathbf{W}, \mathbf{A})$ satisfies the Gentzen rules for every $x \in W, z \in W^{\prime}$ and $a, b \in A$, then $e(a):=\gamma(\{a\})$ defines a homomorphism $e: \mathbf{A} \longrightarrow \mathbf{W}^{+}$.

(2) Furthermore, if $a N b$ implies $a \leq_{\mathbf{A}} b$ for every $a, b \in A$, then $e$ is an embedding.

When A is bounded, the homomorphism $e$ preserves both the least and greatest elements. It should be remarked that Lemma 4.4 actually holds for much more general situations. For instance, $\mathbf{A}$ can be an arbitrary, even 


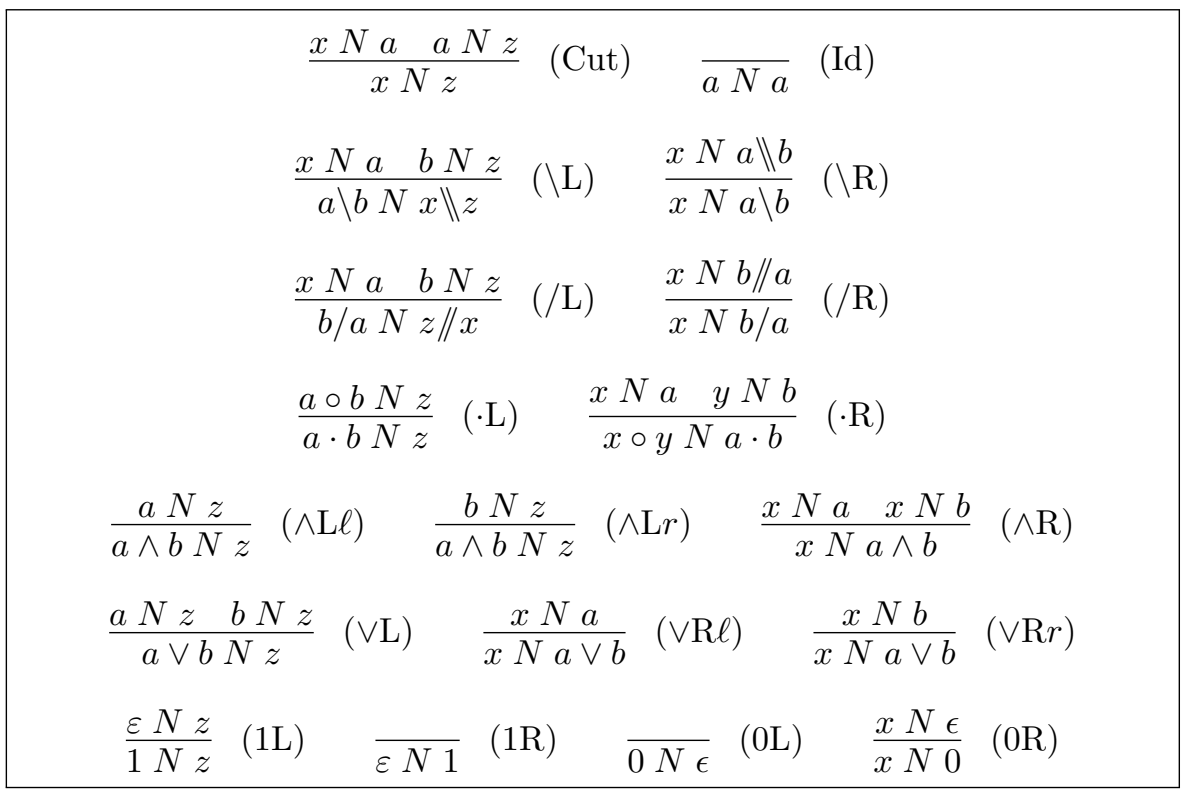

Figure 1. Gentzen rules

partial, algebra in the language of $\mathrm{FL}$, and $i, i^{\prime}$ need not be injections as long as the Gentzen rules are satisfied.

Since $\left(\mathbf{W}_{\mathbf{A}}, \mathbf{A}\right)$ trivially satisfies the Gentzen rules, we see that $\left(\mathbf{W}_{\mathbf{A}}^{+}, e\right)$ is a completion of $\mathbf{A}$. Moreover, it is join-dense and meet-dense since

$$
\begin{aligned}
X & =\bigcup_{\gamma}\{\gamma(a): a \in X\}=\bigcup_{\gamma}\{e(a): e(a) \subseteq X\} \\
& =\bigcap\left\{a^{\triangleleft}: X N a\right\}=\bigcap\{e(a): X \subseteq e(a)\}
\end{aligned}
$$

holds for every Galois-closed set $X$. The last equality holds because $e(a)=$ $a^{\triangleright \triangleleft}=a^{\triangleleft}$ and $X N a$ iff $X \subseteq a^{\triangleleft}$.

Corollary 4.5. $\left(\mathbf{W}_{\mathbf{A}}^{+}, e\right)$ is a MacNeille completion of $\mathbf{A}$. Hence, for every $\mathrm{x} \subseteq\{\mathrm{e}, \mathrm{c}, \mathrm{i}, \mathrm{o}\}$, every chain $\mathbf{A} \in \mathrm{FL}_{\mathrm{x}}^{\ell}$ has a MacNeille completion in $\mathrm{FL}_{\mathrm{x}}^{\ell}$.

The varieties $\mathrm{FL}_{\mathrm{x}}^{\ell}$ with $\mathrm{x} \subseteq\{\mathrm{e}, \mathrm{c}, \mathrm{i}, \mathrm{o}\}$ are just a few examples. We will see in Section 8 that the same holds for many more subvarieties of FL.

\section{Densification of integral FL chains}

Residuated frames are useful not just for completion, but also for densification. In this section, we prove the densifiability of $\mathrm{FL}_{\mathrm{x}}^{\ell}$ with $\{\mathrm{i}\} \subseteq \mathrm{x} \subseteq\{\mathrm{e}, \mathrm{c}, \mathrm{i}, \mathrm{o}\}$ by using residuated frames. Our proof gives a rationale behind the concrete definition of $\mathbf{A}^{p}$ in Section 3, and moreover serves as a warm-up before the more involved case of (non-integral) commutative FL chains in the next section. 
Let us fix an integral FL chain $\mathbf{A}$, a gap $(g, h)$ in it, and a new element $p$. Our purpose is to define a residuated frame whose dual algebra fills the gap $(g, h)$ by $p$.

We define a frame $\mathbf{W}_{\mathbf{A}}^{p}=\left(W, W^{\prime}, N, \circ, \varepsilon, \epsilon\right)$ as follows.

- $(W, \circ, \varepsilon)$ is the free monoid generated by $A \cup\{p\}$.

- $W^{\prime}:=A \cup\{p\}, \epsilon:=0 \in A$. $N$ is defined below.

Thus, each element $x \in W$ is a finite sequence of elements from $A \cup\{p\}$. We denote by $A^{*}$ the subset of $W$ that consists of finite sequences of elements from $A$ (without any occurrence of $p$ ). Also, given $x \in W$, we denote by $\bar{x}$ the product (in $\mathbf{A}$ ) of all elements of $x$ where $p$ is replaced by $h$. For instance, if $x=p a p b \in W$ with $a, b \in A$, then $\bar{x}=h a h b \in A$.

Let us now define $N$. Under the intuition that $g<p<h$ should hold and $N$ should be an extension of $\leq_{\mathbf{A}}$, it is natural to require that $a N p$ iff $a \leq g$, and $p N a$ iff $h \leq a$ for every $a \in A$. We also require that $p N p$. The definition below embodies these requirements. For every $x \in W$ and $a \in A$ :

$$
\begin{array}{ll}
x N a \Longleftrightarrow \bar{x} \leq_{\mathbf{A}} a, & \\
x N p \Longleftrightarrow \bar{x} \leq_{\mathbf{A}} g & \text { (if } \left.x \in A^{*}\right), \\
x N p \text { always holds } & \text { (otherwise). }
\end{array}
$$

As explained in Section 4, the frame $\mathbf{W}_{\mathbf{A}}^{p}$ induces a residuated frame $\tilde{\mathbf{W}}_{\mathbf{A}}^{p}$.

Notice that since $\mathbf{A}$ is an integral chain, $\mathbf{W}_{\mathbf{A}}^{p}$ satisfies

$$
\frac{u v N z}{u x v N z}
$$

for every $u, x, v \in W$ and $z \in W^{\prime}$. Hence, $\tilde{\mathbf{W}}_{\mathbf{A}}^{p}$ satisfies the rule $\left(i^{N}\right)$, so the dual algebra $\tilde{\mathbf{W}}_{\mathbf{A}}^{p+}$ is integral by Lemma 4.3.

To have a closer look at the residuated frame $\tilde{\mathbf{W}}_{\mathbf{A}}^{p}$, it is convenient to partition the set $\tilde{W}^{\prime}=W \times W^{\prime} \times W$ into three, in accordance with the case distinctions in the definition of $N$ :

$$
\begin{aligned}
& \tilde{W}_{1}^{\prime}:=\left\{(u, a, v) \in \tilde{W}^{\prime}: a \in A \text { and } u, v \in W\right\}, \\
& \tilde{W}_{2}^{\prime}:=\left\{(u, p, v) \in \tilde{W}^{\prime}: u, v \in A^{*}\right\}, \\
& \tilde{W}_{3}^{\prime}:=\left\{(u, p, v) \in \tilde{W}^{\prime}: u \notin A^{*} \text { or } v \notin A^{*}\right\} .
\end{aligned}
$$

Just as we associated an element $\bar{x} \in A$ to each $x \in W$, we associate an element $\bar{z} \in A$ to each $z \in \tilde{W}^{\prime}$ as follows:

$$
\bar{z}:= \begin{cases}\bar{u} \backslash a / \bar{v}, & \text { if } z=(u, a, v) \in \tilde{W}_{1}^{\prime} \\ \bar{u} \backslash g / \bar{v}, & \text { if } z=(u, p, v) \in \tilde{W}_{2}^{\prime} \\ 1, & \text { if } z=(u, p, v) \in \tilde{W}_{3}^{\prime} .\end{cases}
$$

Finally, we define $A^{\circ}:=\tilde{W}_{1}^{\prime} \cup \tilde{W}_{3}^{\prime}$. A pair $(x, z) \in W \times \tilde{W}^{\prime}$ is said to be stable if either $x \in A^{*}$ or $z \in A^{\circ}$. We also say that a statement $x \tilde{N} z$ is stable if $(x, z)$ is. 
The following lemma explains why we have defined the sets $A^{*}, A^{\circ}$, and the concept of stability.

\section{Lemma 5.1.}

(1) If $(x, z)$ is stable, then $x \tilde{N} z$ iff $\bar{x} \leq_{\mathbf{A}} \bar{z}$. If not, $x \tilde{N} z$ always holds.

(2) If $x \notin A^{*}$, then $\bar{x} \leq_{\mathbf{A}} h$.

(3) If $z \notin A^{\circ}$, then $g \leq_{\mathbf{A}} \bar{z}$.

Proof. (1): When $z=(u, a, v) \in \tilde{W}_{1}^{\prime}$, we have $x \tilde{N} z$ iff $u x v N a$ iff $\overline{u x v} \leq a$ iff $\bar{x} \leq \bar{z}$. When $z=(u, p, v) \in \tilde{W}_{3}^{\prime}$, both $u x v N p$ and $\bar{x} \leq 1=\bar{z}$ hold. When $z=(u, p, v) \in \tilde{W}_{2}^{\prime}$ and $x \in A^{*}, x \tilde{N} z$ iff $u x v N p$ iff $\overline{u x v} \leq g$ iff $\bar{x} \leq \bar{z}$. When $z \in \tilde{W}_{2}^{\prime}$ and $x \notin A^{*}, x \tilde{N} z$ always holds.

$(2): x \notin A^{*}$ means that the sequence $x$ contains an occurrence of $p$, which is interpreted by $h$. Hence, the claim holds by integrality.

(3): This is proved in a similar way.

Lemma 5.2. $\tilde{\mathbf{W}}_{\mathbf{A}}^{p}$ satisfies the rule $\left(\operatorname{com}^{N}\right)$. Hence $\tilde{\mathbf{W}}_{\mathbf{A}}^{p+}$ is a chain.

Proof. We verify

$$
\frac{x \tilde{N} z \text { and } y \tilde{N} w}{x \tilde{N} w \text { or } y \tilde{N} z}\left(\operatorname{com}^{N}\right) .
$$

In case at least one of the conclusions is not stable, $\left(\mathrm{com}^{N}\right)$ immediately holds by Lemma 5.1(1). Notice that this is always the case when both premises $x \tilde{N} z$ and $y \tilde{N} w$ are not stable. Hence, we only need to consider the cases when both conclusions are stable and either both or only one of the premises is stable.

(i) If both premises $x \tilde{N} z$ and $y \tilde{N} w$ are stable, $\left(\operatorname{com}^{N}\right)$ boils down to

$$
\bar{x} \leq \bar{z} \text { and } \bar{y} \leq \bar{w} \Longrightarrow \bar{x} \leq \bar{w} \text { or } \bar{y} \leq \bar{z},
$$

that holds by the communication property in $\mathbf{A}$.

(ii) Assume only one premise is stable. For instance, let y $\tilde{N} w$ be stable and $x \tilde{N} z$ not stable, so that $x \notin A^{*}$ and $z \notin A^{\circ}$. We have either $\bar{y} \leq g$ or $h \leq \bar{y}$ since $(g, h)$ is a gap. If $\bar{y} \leq g$, then $\bar{y} \leq g \leq \bar{z}$ by Lemma $5.1(3)$, so the right conclusion holds. If $h \leq \bar{y}$, Lemma 5.1(2) and the right premise implies $\bar{x} \leq h \leq \bar{y} \leq \bar{w}$, so the left conclusion holds. The case where $y \tilde{N} w$ is not stable and $x \tilde{N} z$ is stable is symmetrical.

For the next lemma, we consider injections $i, i^{\prime}$ from $A$ to $W$ and $W^{\prime}$ given by $i(a):=a \in W$ and $i^{\prime}(a):=(\varepsilon, a, \varepsilon) \in \tilde{W}^{\prime}$, and identify $a$ with $i(a)$ and $i^{\prime}(a)$.

Lemma 5.3. ( $\left.\tilde{\mathbf{W}}_{\mathbf{A}}^{p}, \mathbf{A}\right)$ satisfies all Gentzen rules. Moreover, a $\tilde{N} b \mathrm{im}$ plies $a \leq_{\mathbf{A}} b$ for every $a, b \in A$. Hence, $e(a):=\gamma(a)$ is an embedding of $\mathbf{A}$ into $\tilde{\mathbf{W}}_{\mathbf{A}}^{p+}$.

Proof. Observe that all Gentzen rules (Figure 1, where $N$ is replaced by $\tilde{N}$ ) have stable premises. If the conclusion is also stable, then the claim follows from the premises by Lemma 5.1(1). Otherwise, (as may happen for the rule (Cut)), the conclusion holds automatically. 
Lemma 5.4. Let $e$ be the embedding of $\mathbf{A}$ into $\tilde{\mathbf{W}}_{\mathbf{A}}^{p+}$ in Lemma 5.3. The following hold.

(1) For every $x \in A \cup\{p\}, e(x)=x^{\triangleright \triangleleft}=x^{\triangleleft}$.

(2) $e(g) \subsetneq e(p) \subsetneq e(h)$.

Proof. (1): Suppose that $x=a \in A$. We have $a \in a^{\triangleleft}$ by (Id). Hence, $a^{\triangleright \triangleleft} \subseteq a^{\triangleleft}$. To show the other inclusion, let $y \in a^{\triangleleft}$ and $z \in a^{\triangleright}$. Then $y \tilde{N} a$ and $a \tilde{N} z$, so $y \tilde{N} z$ by (Cut). This shows that $a^{\triangleleft} \subseteq a^{\triangleright \triangleleft}$.

For $x=p$, the above reasoning shows that it is sufficient to verify (Id) and (Cut) for $p$, too:

$$
\overline{p \tilde{N} p}(\mathrm{Id}) \quad \frac{x \tilde{N} p p \tilde{N} z}{x \tilde{N} z} \text { (Cut) }
$$

Here, we identify $p$ on the right-hand side with $(\varepsilon, p, \varepsilon) \in \tilde{W}^{\prime}$. (Id) is obvious. For (Cut), if the conclusion is unstable, it holds automatically. Otherwise, we distinguish three cases. If $x \in A^{*}$ and $z \notin A^{\circ}$, Lemma 5.1(3) and the left premise (which is stable) imply $\bar{x} \leq g \leq \bar{z}$. If $x \notin A^{*}$ and $z \in A^{\circ}$, Lemma $5.1(2)$ and the right premise (which is stable) imply $\bar{x} \leq h \leq \bar{z}$. If $x \in A^{*}$ and $z \in A^{\circ}$, we have $\bar{x} \leq g<h \leq \bar{z}$.

(2): We have $g \tilde{N} p$ and $p \tilde{N} h$, so $g \in p^{\triangleleft}$ and $p \in h^{\triangleleft}$, which imply $e(g) \subseteq e(p) \subseteq e(h)$ by (1). On the other hand, we have neither $p \tilde{N} g$ nor $h \tilde{N} p$ (that would mean $h \leq g$ ). Hence, the two inclusions are strict.

We have proved that the chain $\tilde{\mathbf{W}}_{\mathbf{A}}^{p+}$ fills the gap $(g, h)$ of $\mathbf{A}$. Clearly, $\tilde{\mathbf{W}}_{\mathbf{A}}^{p}$ satisfies $\left(e^{N}\right),\left(c^{N}\right)$, and $\left(o^{N}\right)$ whenever A satisfies $(e),(c)$, and $(o)$. Hence, by Lemma 4.3, Corollary 4.5, and Proposition 2.3, we conclude the following.

Theorem 5.5. $\mathrm{FL}_{\mathrm{x}}^{\ell}$ with $\{\mathrm{i}\} \subseteq \mathrm{x} \subseteq\{\mathrm{e}, \mathrm{c}, \mathrm{i}, \mathrm{o}\}$ is densifiable and standard complete.

Thus, we have given a uniform proof for the standard completeness of Gödel logic $\left(\mathrm{FL}_{\text {ecio }}^{\ell}\right)$, monoidal t-norm logic $\left(\mathrm{FL}_{\text {eio }}^{\ell}\right)$, and its noncommutative counterpart $\left(\mathrm{FL}_{\mathrm{io}}^{\ell}\right)[23,24]$.

The structure of $\tilde{\mathbf{W}}_{\mathbf{A}}^{p+}$. We have obtained a chain $\tilde{\mathbf{W}}_{\mathbf{A}}^{p+}$ filling a gap of $\mathbf{A}$, but we have not yet seen what kind of chain it is. By looking into its structure, it turns out that it is just a MacNeille completion of the chain $\mathbf{A}^{p}$ presented in Section 3.

We will show that the restriction of $\tilde{\mathbf{W}}_{\mathbf{A}}^{p+}$ to $e[A] \cup\{\gamma(p)\}$ forms a subalgebra by giving a concrete description. To simplify the notation, we write

$$
\hat{x}:=\gamma(x)=x^{\triangleright \triangleleft}=x^{\triangleleft}
$$

for every $x \in A \cup\{p\}$ (cf. Lemma 5.4(1)), and $\hat{x} \cdot \hat{y}:=\hat{x} \circ_{\gamma} \hat{y}$. The lattice structure of $e[A] \cup\{\hat{p}\}$ is already clear (cf. Lemma 5.4(2)). Moreover, since $e(a)=\hat{a}$ is an embedding, we have $\hat{a} \star \hat{b}=\widehat{a \star b}$ for every $a, b \in A$ and $\star \in\{\cdot, \backslash, /\}$. Hence, it is sufficient to determine the operations $\cdot, \backslash, /$ applied to $\hat{a}$ and $\hat{p}$. 
Proposition 5.6. For every $a \in A$, we have:

$\hat{p} \cdot \hat{p}:=\hat{p}$ if $h^{2}=h$, and $\hat{p} \cdot \hat{p}:=\hat{h}^{2}$, otherwise;

$\hat{a} \cdot \hat{p}:=\hat{p}$ if $a h=h$, and $\hat{a} \cdot \hat{p}:=\widehat{a h}$, otherwise;

$\hat{p} \backslash \hat{p}:=\hat{1}$, and $\hat{p} \backslash \hat{a}:=\widehat{h \backslash a}$;

$\hat{a} \backslash \hat{p}:=\hat{p}$ if $a h=h$, and $\hat{a} \backslash \hat{p}:=\widehat{a \backslash g}$, otherwise.

Similar equalities hold for $\hat{p} \cdot \hat{a}, \hat{p} / \hat{p}, \hat{a} / \hat{p}$ and $\hat{p} / \hat{a}$. Hence, the restriction of $\tilde{\mathbf{W}}_{\mathbf{A}}^{p+}$ to $e[A] \cup\{\hat{p}\}$ forms a subalgebra that is isomorphic to $\mathbf{A}^{p}$, and $\tilde{\mathbf{W}}_{\mathbf{A}}^{p+}$ is its MacNeille completion.

Proof. Notice that $\hat{x} \cdot \hat{y}=\gamma(\gamma(x) \circ \gamma(y))=(x y)^{\triangleright \triangleleft}$. Hence, to see the equivalence between $\hat{x} \cdot \hat{y}$ and $\hat{u}$, it is sufficient to check $(x y)^{\triangleright}=u^{\triangleright}$, which holds exactly when $x y \tilde{N} z$ iff $u \tilde{N} z$ for every $z \in \tilde{W}^{\prime}$.

If $z \in A^{\circ}$, stability implies

- $p p \tilde{N} z$ iff $h^{2} \leq \bar{z}$ iff $h \leq \bar{z}$ iff $p \tilde{N} z$ (when $h^{2}=h$ ),

- $p p \tilde{N} z$ iff $h^{2} \leq \bar{z}$ iff $h^{2} \tilde{N} z$ (when $h^{2} \leq g$ ),

- ap $\tilde{N} z$ iff $a h \leq \bar{z}$ iff $h \leq \bar{z}$ iff $p \tilde{N} z$ (when $a h=h$ ),

- ap $\tilde{N} z$ iff $a h \leq \bar{z}$ iff $a h \tilde{N} z$ (when $a h \leq g$ ).

If $z \notin A^{\circ}$, both sides of the above four hold by Lemma 5.1(1) and (3).

To prove the equalities for $\backslash$, notice that $\hat{w}=w^{\triangleleft}$ and $\hat{x} \backslash \hat{z}=x^{\triangleright \triangleleft \backslash} \backslash z^{\triangleleft}=$ $\{x\} \backslash z^{\triangleleft}$ for every $w, x, z \in A \cup\{p\}$. Hence, to see $\hat{x} \backslash \hat{z}=\hat{w}$, it is sufficient to check that $x y \tilde{N} z$ iff $y \tilde{N} w$ for every $y \in W$.

We have $\hat{p} \backslash \hat{p}=\hat{1}$ and $\hat{p} \backslash \hat{a}=\widehat{h \backslash a}$ since

- both py $\tilde{N} p$ and $y \tilde{N} 1$ hold,

- $p y \tilde{N} a$ iff $h \bar{y} \leq a$ iff $\bar{y} \leq h \backslash a$ iff $y \tilde{N} h \backslash a$.

For the equality for $\hat{a} \backslash \hat{p}$, we distinguish two cases. If $y \in A^{*}$, stability implies

- $a y \tilde{N} p$ iff $a \bar{y} \leq g$ iff $\bar{y} \leq g$ iff $y \tilde{N} p$ (when $a h=h$ ). To see the second equivalence, $\bar{y} \leq g$ obviously implies $a \bar{y} \leq g$. Conversely, suppose that $\bar{y} \leq g$ does not hold. Then $h \leq \bar{y}$, so $h=a h \leq a \bar{y}$. Hence, $a \bar{y} \leq g$ does not hold.

- $a y \tilde{N} p$ iff $a \bar{y} \leq g$ iff $\bar{y} \leq a \backslash g$ iff $y \tilde{N} a \backslash g$ (when $a h \leq g$ ).

If $y \notin A^{*}$, both sides of the above two hold. In particular, $y \tilde{N} a \backslash g$ holds since $a \bar{y} \leq a h \leq g$ by Lemma 5.1(2), so $\bar{y} \leq a \backslash g$.

Finally, $\tilde{\mathbf{W}}_{\mathbf{A}}^{p+}$ is a MacNeille completion of $\mathbf{A}^{p}$ since

$$
e[A] \cup\{\hat{p}\}=\{\hat{x}: x \in W\}=\left\{z^{\triangleleft}: z \in W^{\prime}\right\},
$$

and any Galois-closed set $X$ is both a join of elements from the second set and a meet of elements from the third set (cf. (*) in Section 4).

One might call into question the significance of our construction based on residuated frames, since the resulting algebra admits a much simpler presentation as given in Section 3. Our justifications are as follows.

- Our method has a heuristic value, as it provides a general recipe how to 
find a chain that fills a gap. In essence, it amounts to a combinatorial task of finding a residuated frame satisfying $\left(\mathrm{com}^{N}\right)$ and Gentzen rules. Once such a frame has been found, we are done. See the next section for another application of our method.

- To prove standard completeness, we usually need to show both densifiability and closure under MacNeille completions (Proposition 2.3). Since residuated frames unify the two tasks to a large extent, the method may in fact be considered quite economical.

- Residuated frames are intimately connected to the sequent calculus in proof theory, as one can see in the Gentzen rules of Figure 1. This allows us to translate various proof theoretic arguments into algebraic ones. Indeed, our construction was inspired by a proof theoretic argument for standard completeness: first introduce the density rule in the hypersequent calculus, which enforces the intended algebraic models to be dense chains, and then show that it can be eliminated from a given proof, thus relating dense chains with nondense ones $[1,26,11,12,4]$. Further references on the subject can be found in $[27,25]$. Our frame construction precisely mirrors the way that the density rule is eliminated in $[12,4]$. It is amazing that such a proof theoretic argument, devised independently of algebraic considerations, translates into an algebraic one quite smoothly. It suggests a deep connection between proof theory and algebra, perhaps much deeper than usually believed. Finding such a connection is the main goal of our long-term project: algebraic proof theory for substructural logics [9, 10].

\section{Densification of commutative FL chains}

We now turn to another class of chains: commutative FL chains. The class of bounded commutative FL chains provides a general algebraic semantics for so-called uninorm logic, which is known to be standard complete [26]. However, all the known proofs of this fact are proof theoretic, based on elimination of the density rule in a hypersequent calculus. In this section, we translate the proof theoretic argument into an algebraic one based on residuated frames. This gives rise to a first algebraic proof of standard completeness for uninorm logic.

Let $\mathbf{A}$ be a commutative FL chain with a gap $(g, h)$ and $p$ a new element. We again build a residuated frame whose dual algebra fills the gap $(g, h)$. Although we could define $W$ as before, we can exploit commutativity to simplify the construction.

We define a frame $\mathbf{W}_{\mathbf{A}}^{p}:=\left(W, W^{\prime}, N, \circ, \varepsilon, \epsilon\right)$ as follows:

- $W:=A \times \mathbb{N}$. Each element $(a, m) \in W$ is denoted by $a p^{m}$ as if it were a polynomial in the variable $p$. We identify $A$ with the subset $\left\{a p^{0}: a \in A\right\}$ of $W$.

- $a p^{m} \circ b p^{n}:=(a b) p^{m+n}$ and $\varepsilon:=1=1 p^{0}$. 
- $W^{\prime}:=A \cup\{p\}$ and $\epsilon:=0 \in A$.

- There are three types of elements in $W \times W^{\prime}:\left(a p^{n}, b\right),(a, p)$, and $\left(a p^{n+1}, p\right)$ with $a, b \in A$ and $n \in \mathbb{N}$. $N$ is defined accordingly:

$$
\begin{aligned}
a p^{n} N b & \Longleftrightarrow a h^{n} \leq_{\mathbf{A}} b, \\
a N p & \Longleftrightarrow a \leq_{\mathbf{A}} g, \\
a p^{n+1} N p & \Longleftrightarrow a h^{n} \leq_{\mathbf{A}} 1 .
\end{aligned}
$$

Notice that this is compatible with the previous definition. In particular, $a p^{n+1} N p$ always holds if $\mathbf{A}$ is integral. As before, the frame $\mathbf{W}_{\mathbf{A}}^{p}$ induces a residuated frame $\tilde{\mathbf{W}}_{\mathbf{A}}^{p}:=\left(W, \tilde{W}^{\prime}, \tilde{N}, \circ, \varepsilon,(\varepsilon, \epsilon)\right)$. Because of commutativity, the definitions of $\tilde{W}^{\prime}$ and $\tilde{N}$ are slightly simplified:

$$
\tilde{W}^{\prime}:=W \times W^{\prime}, \quad x \tilde{N}(y, z) \text { iff } x \circ y N z .
$$

As in the integral case, the set $\tilde{W}^{\prime}$ can be partitioned into three:

$$
\begin{aligned}
& \tilde{W}_{1}^{\prime}:=\left\{\left(a p^{n}, b\right): a, b \in A, n \geq 0\right\}, \\
& \tilde{W}_{2}^{\prime}:=\{(a, p): a \in A\}, \\
& \tilde{W}_{3}^{\prime}:=\left\{\left(a p^{n+1}, p\right): a \in A, n \geq 0\right\} .
\end{aligned}
$$

Elements of $W, \tilde{W}^{\prime}$ are interpreted by elements of $A$. For $x=a p^{n} \in W$, let $\bar{x}:=a h^{n} \in A$. For $z \in \tilde{W}^{\prime}$, we define

$$
\bar{z}:= \begin{cases}a h^{n} \rightarrow b, & \text { if } z=\left(a p^{n}, b\right) \in \tilde{W}_{1}^{\prime} \\ a \rightarrow g, & \text { if } z=(a, p) \in \tilde{W}_{2}^{\prime} \\ a h^{n} \rightarrow 1, & \text { if } z=\left(a p^{n+1}, p\right) \in \tilde{W}_{3}^{\prime} .\end{cases}
$$

As before, $A^{\circ}:=\tilde{W}_{1}^{\prime} \cup \tilde{W}_{3}^{\prime}$. A pair $(x, z) \in W \times \tilde{W}^{\prime}$ is stable if either $x \in A$ or $z \in A^{\circ}$. Similarly to Lemma 5.1(1), we have the following result.

Lemma 6.1. If $(x, z)$ is stable, then $x \tilde{N} z$ iff $\bar{x} \leq_{\mathbf{A}} \bar{z}$.

Proof. When $x \in A$ and $z=(a, p) \in \tilde{W}_{2}^{\prime}$, we have $x \tilde{N} z$ iff $x a N p$ iff $x a \leq g$ iff $x \leq a \rightarrow g$ iff $\bar{x} \leq \bar{z}$.

When $x=x^{\prime} p^{m}$ and $z=\left(a p^{n}, b\right) \in \tilde{W}_{1}^{\prime}$, we have $x \tilde{N} z$ iff $x^{\prime} a p^{m+n} N b$ iff $x^{\prime} a h^{m+n} \leq b$ iff $x^{\prime} h^{m} \leq a h^{n} \rightarrow b$ iff $\bar{x} \leq \bar{z}$.

When $x=x^{\prime} p^{m}$ and $z=\left(a p^{n+1}, p\right) \in \tilde{W}_{3}^{\prime}$, we have $x \tilde{N} z$ iff $x^{\prime} a p^{m+n+1} N p$ iff $x^{\prime} a h^{m+n} \leq 1$ iff $x^{\prime} h^{m} \leq a h^{n} \rightarrow 1$ iff $\bar{x} \leq \bar{z}$.

Lemma 6.2. $\tilde{\mathbf{W}}_{\mathbf{A}}^{p}$ satisfies the rule $\left(\operatorname{com}^{N}\right)$.

Proof. We verify

$$
\frac{x \tilde{N} z \text { and } y \tilde{N} w}{x \tilde{N} w \text { or } y \tilde{N} z}\left(\operatorname{com}^{N}\right) .
$$

(i): If $x, y \in A$ or $w, z \in A^{\circ}$, all the pairs $\{x, y\} \times\{z, w\}$ are stable. By Lemma 6.1 , the rule boils down to

$$
\bar{x} \leq \bar{z} \text { and } \bar{y} \leq \bar{w} \Longrightarrow \bar{x} \leq \bar{w} \text { or } \bar{y} \leq \bar{z},
$$


which holds by the communication property in $\mathbf{A}$.

(ii): Suppose that $w \notin A^{\circ}$ and $z \notin A^{\circ}$. Then $w=(a, p)$ and $z=(b, p)$, so that $\left(\operatorname{com}^{N}\right)$ becomes

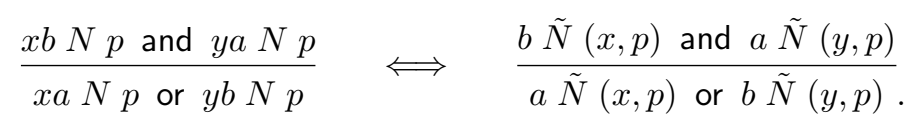

Since $a, b \in A$, this reduces to the case (i).

(iii): Suppose that $w \in A^{\circ}$ and $z \notin A^{\circ}$. We write $w=\left(w_{1}, w_{2}\right)$ and $z=(a, p)$. There are three subcases.

First, suppose that $x, y \notin A$. Then we may write $x=x^{\prime} p$ and $y=y^{\prime} p$, so that $\left(\operatorname{com}^{N}\right)$ becomes

$$
\frac{x^{\prime} \tilde{N}(p a, p) \text { and } y^{\prime} \tilde{N}\left(p w_{1}, w_{2}\right)}{x^{\prime} \tilde{N}\left(p w_{1}, w_{2}\right) \text { or } y^{\prime} \tilde{N}(p a, p) .}
$$

Since $(p a, p),\left(p w_{1}, w_{2}\right) \in A^{\circ}$, this reduces to the case (i).

Second, suppose that $x \in A$ and $y \notin A$, so that we may write $y=y^{\prime} p$. Notice that $x \tilde{N} z$ iff $x a N p$ iff $x a \leq g$. Also, $y \tilde{N} z$ iff $y^{\prime} p a N p$ iff $\bar{y}^{\prime} a \leq 1$. Thus, what we have to check is

$$
x a \leq g \text { and } \bar{y} \leq \bar{w} \Longrightarrow x \leq \bar{w} \text { or } \bar{y}^{\prime} a \leq 1 .
$$

By the communication property, the premises imply either $x \leq \bar{w}$ or $\bar{y} a \leq g$. If $x \leq \bar{w}$, we are done. Otherwise, we have $\bar{y}^{\prime} h a=\bar{y} a \leq g$, so $\bar{y}^{\prime} a \leq h \rightarrow g<1$ (since $g<h$ ). So, we are done.

Finally, suppose that $x \notin A$ and $y \in A$, so that we may write $x=x^{\prime} p$. Note that $x \tilde{N} z$ iff $x^{\prime} p a N p$ iff $\bar{x}^{\prime} a \leq 1$. Also, $y \tilde{N} z$ iff $y a N p$ iff $y a \leq g$. Thus, what we have to check is

$$
\bar{x}^{\prime} a \leq 1 \text { and } y \leq \bar{w} \Longrightarrow \bar{x} \leq \bar{w} \text { or } y a \leq g .
$$

If $y a \leq g$, we are done. Otherwise, $h \leq y a$. Hence, together with the premises, we obtain $\bar{x}=\bar{x}^{\prime} h \leq \bar{x}^{\prime} y a \leq y \leq \bar{w}$.

Lemma 6.3. ( $\left.\tilde{\mathbf{W}}_{\mathbf{A}}^{p}, \mathbf{A}\right)$ satisfies all the Gentzen rules, and moreover a $N b$ implies $a \leq_{\mathbf{A}} b$ for every $a, b \in A$. Hence, $e(a):=\gamma(a)$ is an embedding of $\mathbf{A}$ into $\tilde{\mathbf{W}}_{\mathbf{A}}^{p+}$.

Proof. As in the proof of Lemma 5.3, notice that all Gentzen rules except (Cut) have stable premises and conclusion. Hence, we only have to check the (Cut) rule

$$
\frac{x \tilde{N} a a \tilde{N} z}{x \tilde{N} z} \quad \text { (Cut), }
$$

where $(x, z)$ is unstable. We may write $x=x^{\prime} p$ and $z=(b, p)$. By noting that $x \tilde{N} z$ iff $x^{\prime} p b N p$ iff $\bar{x}^{\prime} b \leq 1$, this amounts to

$$
\bar{x}^{\prime} h \leq a \text { and } a b \leq g \quad \Longrightarrow \quad \bar{x}^{\prime} b \leq 1 \text {. }
$$

Now the premises imply $\bar{x}^{\prime} h b \leq g$, so $\bar{x}^{\prime} b \leq h \rightarrow g<1$. 
Lemma 6.4. Let $e$ be the embedding of $\mathbf{A}$ into $\tilde{\mathbf{W}}_{\mathbf{A}}^{p+}$ in Lemma 6.3. The following hold.

(1) For every $z \in W^{\prime}, e(z)=z^{\triangleright \triangleleft}=z^{\triangleleft}$.

(2) $e(g) \subsetneq e(p) \subsetneq e(h)$.

Proof. In view of the proof of Lemma 5.4, it is sufficient to show that (Id) and (Cut) hold for $p$. For (Cut),

$$
\frac{x \tilde{N} p p \tilde{N} z}{x \tilde{N} z} \text { (Cut). }
$$

If $x \in A$ and $z \in A^{\circ}$, then all of $(x, z),(x, p)$, and $(p, z)$ are stable. Hence, the premises imply $\bar{x} \leq g<h \leq \bar{z}$.

If $x \notin A$ and $z \in A^{\circ}$, we may write $x=x^{\prime} p$. The premises amount to $\bar{x}^{\prime} \leq 1$ and $h \leq \bar{z}$, so we obtain $\bar{x}=\bar{x}^{\prime} h \leq \bar{z}$.

If $x \notin A$ and $z \notin A^{\circ}$, we may write $x=x^{\prime} p$ and $z=(a, p)$. The premises amount to $\bar{x}^{\prime} \leq 1$ and $a \leq 1$, so we obtain $\bar{x}^{\prime} a \leq 1$.

Finally, if $x \in A$ and $z \notin A^{\circ}$, we may write $z=(a, p)$. The premises amount to $x \leq g$ and $a \leq 1$, so we obtain $x a \leq g$.

In any case, we obtain the conclusion $x \tilde{N} z$.

We have proved that the chain $\tilde{\mathbf{W}}_{\mathbf{A}}^{p+}$ fills the gap $(g, h)$ of $\mathbf{A}$. An alternative construction of this algebra and a finer analysis of its structure are provided in [17]. It is easy to see that $\tilde{\mathbf{W}}_{\mathbf{A}}^{p}$ satisfies $\left(e^{N}\right),\left(i^{N}\right),\left(o^{N}\right)$ whenever $\mathbf{A}$ satisfies $(e),(i),(o)$. Thus, we have the following.

Theorem 6.5. Every variety $\mathrm{FL}_{\mathrm{x}}^{\ell}$ with $\{\mathrm{e}\} \subseteq \mathrm{x} \subseteq\{\mathrm{e}, \mathrm{i}, \mathrm{o}\}$ is densifiable, hence is standard complete.

As we mentioned at the beginning of the section, uninorm logic is complete with respect to the class of bounded commutative FL chains. Since boundedness is clearly preserved by our construction, we have obtained a purely algebraic proof of the standard completeness of uninorm logic [26].

\section{Substructural hierarchy and MacNeille completions}

The rest of this paper is devoted to densification of subvarieties of $F L_{i}^{\ell}$ and $\mathrm{FL}_{\mathrm{e}}^{\ell}$. The concept of substructural hierarchy $[7,8,9,10]$ is useful to deal with those subvarieties systematically.

Definition 7.1. For each $n \geq 0$, the sets $\mathcal{P}_{n}$ and $\mathcal{N}_{n}$ are defined as follows.

(0) $\mathcal{P}_{0}=\mathcal{N}_{0}=$ the set of variables.

(P1) 1 and all terms $t \in \mathcal{N}_{n}$ belong to $\mathcal{P}_{n+1}$.

(P2) If $t, u \in \mathcal{P}_{n+1}$, then $t \vee u, t \cdot u \in \mathcal{P}_{n+1}$.

(N1) 0 and all terms $t \in \mathcal{P}_{n}$ belong to $\mathcal{N}_{n+1}$.

(N2) If $t, u \in \mathcal{N}_{n+1}$, then $t \wedge u \in \mathcal{N}_{n+1}$.

(N3) If $t \in \mathcal{P}_{n+1}$ and $u \in \mathcal{N}_{n+1}$, then $t \backslash u, u / t \in \mathcal{N}_{n+1}$. 


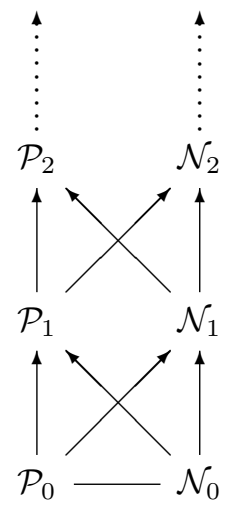

Figure 2. The substructural hierarchy

In other words, $\mathcal{P}_{n}$ and $\mathcal{N}_{n}(n \geq 1)$ are generated by the following BNF grammar:

$$
\begin{array}{ll|l|l|l|ll}
\mathcal{P}_{n} & :=\mathcal{N}_{n-1} & 1 & \mathcal{P}_{n} \vee \mathcal{P}_{n} & \mathcal{P}_{n} \cdot \mathcal{P}_{n}, & \\
\mathcal{N}_{n} & :=\mathcal{P}_{n-1} & 0 & \mathcal{N}_{n} \wedge \mathcal{N}_{n} & \mathcal{P}_{n} \backslash \mathcal{N}_{n} & \mathcal{N}_{n} / \mathcal{P}_{n}
\end{array}
$$

By residuation, any equation $u=v$ can be written as $1 \leq t$. We say that $u=v$ belongs to $\mathcal{P}_{n}\left(\mathcal{N}_{n}\right.$, resp. $)$ if $t$ does.

The classes $\left(\mathcal{P}_{n}, \mathcal{N}_{n}\right)$ constitute the substructural hierarchy (Figure 2). Among those classes, relevant to subsequent arguments, are $\mathcal{N}_{2}$ and $\mathcal{P}_{3}$. The former includes the following:

$$
\begin{aligned}
x y & \leq y x & & (e) \\
x & \leq x x & & (c) \\
x & \leq 1 & & (i) \\
0 & \leq x & & (o) \\
x^{m} & \leq x^{n} & & \text { (knotted axioms, } m \\
1 & \leq \sim(x \wedge \sim x) & & \text { (non-contradiction) }
\end{aligned}
$$

$\mathcal{P}_{3}$ includes the following:

$$
\begin{aligned}
& 1 \leq x \vee \sim x \\
& \text { (excluded middle) } \\
& 1 \leq \sim x \vee \sim \sim x \\
& \text { (weak excluded middle) } \\
& 1 \leq \sim(x \cdot y) \vee(x \wedge y \backslash x \cdot y) \\
& \text { (weak nilpotent minimum) } \\
& 1 \leq \sim(x \cdot y)^{n} \vee\left((x \wedge y)^{n-1} \backslash(x \cdot y)^{n}\right) \\
& \left(\mathrm{wnm}^{n}\right) \\
& 1 \leq\left(x^{n-1} \backslash x \cdot y\right) \vee(y \backslash x \cdot y) \\
& \left(\Omega_{n}\right) \\
& 1 \leq \bigvee_{i=0}^{k}\left(p_{0} \wedge \cdots \wedge p_{i-1} \backslash p_{i}\right) \\
& \text { (bounded width } k \text { ) } \\
& 1 \leq p_{0} \vee\left(p_{0} \backslash p_{1}\right) \vee \cdots \vee\left(p_{0} \wedge \cdots \wedge p_{k-1} \backslash p_{k}\right) \quad \text { (bounded size } k \text { ) }
\end{aligned}
$$


The classes $\mathcal{N}_{2}$ and $\mathcal{P}_{3}$ are intimately related to the classes of structural quasiequations and structural clauses defined below.

Definition 7.2. By a clause, we mean a classical first-order formula of the form

$$
t_{1} \leq u_{1} \text { and } \cdots \text { and } t_{m} \leq u_{m} \Longrightarrow t_{m+1} \leq u_{m+1} \text { or } \cdots \text { or } t_{n} \leq u_{n},(q)
$$

where $t_{i}, u_{i}$ are terms of FL and all variables are assumed to be universally quantified. Each $t_{i} \leq u_{i}(1 \leq i \leq m)$ is called a premise, while each $t_{j} \leq u_{j}$ $(m+1 \leq j \leq n)$ is a conclusion. We say $(q)$ is a quasiequation if $n=m+1$. It is structural if $t_{1}, \ldots, t_{n}$ are products of variables (including the empty product 1 ) and $u_{1}, \ldots, u_{n}$ are either a variable or 0 . Given a structural clause $(q)$, let $L(q)$ be the set of variables occurring in $t_{m+1}, \ldots, t_{n}$, and $R(q)$ the set of variables occurring in $u_{m+1}, \ldots, u_{n}$. We say $(q)$ is analytic if the following conditions are satisfied:

Separation: $L(q)$ and $R(q)$ are disjoint.

Linearity: Each variable in $L(q) \cup R(q)$ occurs exactly once in the conclusions $t_{m+1} \leq u_{m+1}, \ldots, t_{n} \leq u_{n}$.

Inclusion: Each of $t_{1}, \ldots, t_{m}$ is a product of variables in $L(q)$, while each of $u_{1}, \ldots, u_{m}$ is either a variable in $R(q)$ or 0 .

\section{Theorem 7.3.}

(1) Every equation in $\mathcal{N}_{2}$ is equivalent in integral $F L$ algebras to a set of analytic quasiequations.

(2) Every equation in $\mathcal{P}_{3}$ is equivalent in integral $F L$ chains to a set of analytic clauses.

Proof. (1) is proved in [9]. For (2), we have

$$
\begin{aligned}
\mathbf{A} \models t \vee u=1 & \Longleftrightarrow \mathbf{A} \models(t=1 \text { or } u=1), \\
\mathbf{A} \models t \cdot u=1 & \Longleftrightarrow \mathbf{A} \models(t=1 \text { and } u=1),
\end{aligned}
$$

for every integral FL chain $\mathbf{A}$. Thus, each $\mathcal{P}_{3}$ equation is equivalent to a set of disjunctions of the form $\left(t_{1}=1\right.$ or $\cdots$ or $\left.t_{n}=1\right)$. The rest of the proof proceeds as in $[8,10]$.

Example 7.4. Our running example is the weak nilpotent minimum axiom $1 \leq \sim(x y) \vee(x \wedge y \backslash x y)$ that belongs to $\mathcal{P}_{3}$. It is equivalent in integral $\mathrm{FL}$ chains to

$x y \leq z$ and $x v \leq z$ and $v y \leq z$ and $v v \leq z \Longrightarrow x y \leq 0$ or $v \leq z$. (wnm)

Structural clauses are useful because they can be expressed as rules for residuated frames. Moreover, analytic ones are preserved under the dual algebra construction. To make it more precise, consider a structural clause of the form (q) above, and let $\mathbf{W}=\left(W, W^{\prime}, N, \circ, \varepsilon, \epsilon\right)$ be a residuated frame. We can naturally translate each $t_{i}$ into a term over $(\circ, \varepsilon)$, and each $u_{i}$ into either 
a variable or $\epsilon$. The resulting terms are still denoted by $t_{i}, u_{i}$. Corresponding to the clause $(q)$, we have

$$
t_{1} N u_{1} \text { and } \cdots \text { and } t_{m} N u_{m} \Longrightarrow t_{m+1} N u_{m+1} \text { or } \cdots \text { or } t_{n} N u_{n} .\left(q^{N}\right)
$$

Example 7.5. The clause (wnm) corresponds to the following rule for residuated frames:

$x y N z$ and $x v N z$ and $v y N z$ and $v v N z \Rightarrow x y N \epsilon$ or $v N z$.

$\left(w n m^{N}\right)$

By definition, if an FL algebra A satisfies $(q)$, then the residuated frame $\mathbf{W}_{\mathbf{A}}$ satisfies $\left(q^{N}\right)$. Moreover, Lemma 4.3 generalizes to all analytic clauses.

Theorem 7.6. Let $(q)$ be an analytic clause. If a residuated frame $\mathbf{W}$ satisfies $\left(q^{N}\right)$, then the dual algebra $\mathbf{W}^{+}$satisfies $(q)$.

The correctness of the above theorem should be clear from the example below as well as the case of ( $\mathrm{com}$ ) handled by Lemma 4.3. The case of quasiequations is detailed in [9] and the case of clauses is implicit in [8]; [10] contains a more general result.

Example 7.7. Suppose that $\mathbf{W}$ satisfies $\left(w n m^{N}\right)$. Our goal is to show that $\mathbf{W}^{+}$satisfies $(w n m)$, namely

$X Y \subseteq Z$ and $X V \subseteq Z$ and $V Y \subseteq Z$ and $V V \subseteq Z \Longrightarrow X Y \subseteq \epsilon^{\triangleleft}$ or $V \subseteq Z$ holds for all Galois-closed sets $X, Y, V, Z$. Suppose that neither of the conclusions holds. Then there are $x \in X, y \in Y, v \in V$, and $z \in Z^{\triangleright}$ such that neither $x y \in \in$ nor $v N z$ holds (since $Z=Z^{\triangleright \triangleleft}$ ).

On the other hand, the premises yield $x y N z, x v N z, v y N z$, and $v v N z$, which contradict the assumption that $\mathbf{W}$ satisfies the rule $\left(w_{n m}^{N}\right)$.

Corollary 4.5 and Theorem 7.6 lead to the following general result on MacNeille completions.

Theorem 7.8. Let $\mathrm{V}$ be a variety of FL algebras.

(1) If $\mathrm{V}$ is defined by equations equivalent to analytic quasiequations and $\mathbf{A} \in \mathrm{V}$, then its MacNeille completion belongs to $\mathrm{V}$.

(2) If $\mathrm{V}$ is defined by equations equivalent to analytic clauses (over chains) and $\mathbf{A}$ is a chain in $\mathrm{V}$, then its MacNeille completion belongs to $\mathrm{V}$.

\section{Densification of subvarieties of $F L_{i}^{\ell}$}

We now focus on subvarieties of $\mathrm{FL}_{\mathrm{i}}^{\ell}$ defined by $\mathcal{P}_{3}$ equations. By Theorems 7.3 and 7.8, such varieties are always closed under MacNeille completions (applied to chains). However, there are such varieties that do not admit densification. A typical counterexample is the variety BA of Boolean algebras, 
whose only nontrivial chain is the two element one. Notice that BA is defined by excluded middle $x \vee \neg x=1$ in $\mathcal{P}_{2}$, which is equivalent to

$$
x y \leq z \Longrightarrow x \leq 0 \text { or } y \leq z .
$$

We should rule out such a clause by introducing some criteria. The criteria below are inspired by the proof-theoretical approach in [4], which extends [12].

Before we proceed further, let us make it precise what it means that the specific residuated frame $\tilde{\mathbf{W}}_{\mathbf{A}}^{p}$ defined in Section 5 satisfies $\left(q^{N}\right)$. Recall that an analytic clause $(q)$ is of the form

$$
t_{1} \leq z_{1} \text { and } \cdots \text { and } t_{m} \leq z_{m} \Longrightarrow t_{m+1} \leq z_{m+1} \text { or } \cdots \text { or } t_{n} \leq z_{n} .
$$

For the purpose of this section, it is convenient to write $(q)$ as $P \Longrightarrow C$, where

$$
P:=\left\{t_{1} \leq z_{1}, \ldots, t_{m} \leq z_{m}\right\}, \quad C:=\left\{t_{m+1} \leq z_{m+1}, \ldots, t_{n} \leq z_{n}\right\} .
$$

Recall that each equation in $P$ and $C$ consists of variables $L(q)$ and $R(q)$. To each $x \in L(q)$, we associate an element $x^{\bullet} \in W=(A \cup\{p\})^{*}$ so that each term $t$ is interpreted by $t^{\bullet} \in W$. Likewise, to each $z \in R(q)$ we associate a triple $z^{\bullet} \in \tilde{W}^{\prime}=W \times W^{\prime} \times W$, where $W^{\prime}=A \cup\{p\}$. The interpretations of constants 1, 0 are already fixed: $1^{\bullet}:=\varepsilon \in W$ and $0^{\bullet}:=(\varepsilon, \epsilon, \varepsilon)=(\varepsilon, 0, \varepsilon) \in \tilde{W}^{\prime}$. It is now clear when $\tilde{\mathbf{W}}_{\mathbf{A}}^{p}$ satisfies $\left(q^{N}\right)$. It is true just in case the following holds for each such interpretation $\bullet$ :

$$
\left\{t^{\bullet} \tilde{N} z^{\bullet}: t \leq z \in P\right\} \quad \Longrightarrow\left\{t^{\bullet} \tilde{N} z^{\bullet}: t \leq z \in C\right\} .
$$

Suppose that $t$ is a product of variables: $t=x_{1} \cdots x_{n}$. Then $t^{\bullet} \in A^{*}$ iff $x_{i}^{\bullet} \in A^{*}$ for every $1 \leq i \leq n$. This implies:

$\left(t^{\bullet}, z^{\bullet}\right)$ is stable $\Longleftrightarrow\left(x_{i}^{\bullet}, z^{\bullet}\right)$ is stable for every $1 \leq i \leq n$.

Let us now come back to criteria for densifiability.

Definition 8.1. Let $(q): P \Longrightarrow C$ be an analytic clause. Let us define $D P(q):=\{(x, z): u x v \leq z \in P\}$ and $D C(q):=\{(x, z): u x v \leq z \in C\}$. That is, $D P(q)$ is the set of pairs of variables "connected" by one of the premises, and similarly for $D C(q)$. We say that $(q)$ is anchored if $D P(q) \subseteq D C(q)$.

Clearly, the clause $(e m)$ is not anchored since $D P(e m)=\{(x, z),(y, z)\}$ and $D C(\mathrm{em})=\{(y, z)\}$. On the other hand, any analytic quasiequation is anchored due to the inclusion condition.

Lemma 8.2. Let $\mathbf{A}$ be an integral $F L$ chain with a gap $(g, h)$ and $(q)$ an anchored analytic clause. If $\mathbf{A}$ satisfies $(q)$, then the residuated frame $\tilde{\mathbf{W}}_{\mathbf{A}}^{p}$ satisfies $\left(q^{N}\right)$. In particular, if $\mathbf{A}$ satisfies an analytic quasiequation $(q), \tilde{\mathbf{W}}_{\mathbf{A}}^{p}$ satisfies $\left(q^{N}\right)$.

Proof. Assume that A satisfies an anchored clause $(q)$. Our goal is to verify (*) above for any interpretation $\bullet$. If there is a conclusion $t \leq z \in C$ such that $\left(t^{\bullet}, z^{\bullet}\right)$ is not stable, then we have $t^{\bullet} \tilde{N} z^{\bullet}$ by Lemma 5.1(1), so (*) holds. 
Otherwise, $\left(t^{\bullet}, z^{\bullet}\right)$ is stable for every $t \leq z \in C$, and the same holds for every $t \leq z \in P$ by $(\$)$ and $D P(q) \subseteq D C(q)$. Hence, by Lemma 5.1(1), (*) amounts to $\left\{\bar{t}^{\bullet} \leq_{\mathbf{A}} \bar{z}^{\bullet}: t \leq z \in P\right\} \quad \Longrightarrow \quad\left\{\bar{t}^{\bullet} \leq_{\mathbf{A}} \bar{z}^{\bullet}: t \leq z \in C\right\}$, which holds since A satisfies $(q)$.

The previous lemma does not apply to many clauses. For instance, it does not apply to $(w n m)$ :

$$
x y \leq z, \quad x v \leq z, \quad v y \leq z, \quad v v \leq z \quad \Longrightarrow \quad x y \leq 0 \text { or } v \leq z, \quad(w n m)
$$

since $(x, z),(y, z) \in D P(w n m) \backslash D C(w n m)$. To deal with this and more involved clauses, we need to extend the definition of anchored clause.

In the sequel, we write $t=t\left(x_{1}, \ldots, x_{n}\right)$ to indicate variable occurrences $x_{1}, \ldots, x_{n}$ in term $t$. Then $t\left(y_{1}, \ldots, y_{n}\right)$ denotes the result of substituting $y_{i}$ for $x_{i}$.

Definition 8.3. Let $(q): P \Longrightarrow C$ be an analytic clause. We say that $(q)$ is semi-anchored if for every premise $t \leq z$ in $P$, if $t$ can be written as $t\left(x_{1}, \ldots, x_{n}\right)$, with $\left(x_{i}, z\right) \notin D C(q)(1 \leq i \leq n)$, one of the following holds:

(1) there is a premise $t\left(y_{1}, \ldots, y_{n}\right) \leq z$ in $P$ with $\left(y_{i}, z\right) \in D C(q)$ for every $1 \leq i \leq n$,

(2) there is a premise $t\left(x_{1}, \ldots, x_{n}\right) \leq w$ in $P$ with $\left(x_{i}, w\right) \in D C(q)$ for some $1 \leq i \leq n$,

$\left(t\right.$ may contain a variable $x_{0} \notin\left\{x_{1}, \ldots, x_{n}\right\}$ with $\left(x_{0}, z\right) \notin D C(q)$.)

Notice that checking semi-anchoredness amounts to checking finitely many conditions on the premises and the conclusions of a clause; hence, it is decidable. The PROLOG-system Axiom Calc automates the conversion of equations into analytic clauses in Section 7 and checks whether an analytic clause satisfies a condition similar to (1) in Definition 8.3; see [4]. Condition (2) in Definition 8.3 does not present any particular challenge for further automation. (AxiomCalc is available online at http://www.logic.at/people/lara/ axiomcalc.html.)

Example 8.4. (wnm) is semi-anchored. Note that $D C(w n m)=\{(v, z)\}$. For the first premise $x y \leq z$, the term $x y$ can be written as $t_{1}(x)=t_{2}(y)=$ $t_{3}(x, y)=x y$, so that $t_{1}(v)=v y, t_{2}(v)=x v$, and $t_{3}(v, v)=v v$. In any case, we have premises $t_{1}(v) \leq z, t_{2}(v) \leq z$, and $t_{3}(v, v) \leq z$ with $(v, z) \in D C($ wnm $)$, so the case (1) applies. Similarly for the other premises.

Likewise, we can show that $\left(w n m^{n}\right)$ is equivalent to a semi-anchored clause for every $n$. This conforms to the standard completeness of monoidal t-norm logic with $\left(w_{n m}^{n}\right)$ proved in [4].

Example 8.5. The equations $\left(\Omega_{n}\right)$ are equivalent in integral FL chains to the conjunction of $x^{n-1} \leq x^{n}$ and $\left(x^{n-1} \backslash y\right) \vee(y \backslash x \cdot y)$. $\mathrm{FL}_{e w}$ chains satisfying these equations are called $\Omega\left(S_{n} M T L\right)$ chains and are shown to be densifiable 
in [22]. The equation $\left(\Omega_{3}\right)$ is equivalent to the analytic clause

$$
y x \leq z_{1}, \quad w x \leq z_{1}, \quad y x \leq z_{2}, \quad w x \leq z_{2} \quad \Longrightarrow \quad w y \leq z_{2} \text { or } x \leq z_{1}
$$

This clause is semi-anchored. Note that $D C\left(\Omega_{3}\right)=\left\{\left(w, z_{2}\right),\left(y, z_{2}\right),\left(x, z_{1}\right)\right\}$. For the first premise, $y x$ can be written as $t_{1}(y)=y x$. We have $t_{1}(y) \leq z_{2}$ in the premise with $\left(y, z_{2}\right) \in D C\left(\Omega_{3}\right)$, so that case (2) applies. For the third premise, $y x$ can be written as $t_{2}(x)=y x$. We have $t_{2}(x) \leq z_{1}$ in the premise with $\left(x, z_{1}\right) \in D C\left(\Omega_{3}\right)$. Similarly for the second and fourth premises.

Lemma 8.6. Let $\mathbf{A}$ be an integral $F L$ chain with a gap $(g, h)$ and $(q): P \Longrightarrow C$ a semi-anchored analytic clause. If $\mathbf{A}$ satisfies $(q)$, then $\tilde{\mathbf{W}}_{\mathbf{A}}^{p}$ satisfies $\left(q^{N}\right)$.

Proof. Our goal is again to show that

$$
\left\{t^{\bullet} \tilde{N} z^{\bullet}: t \leq z \in P\right\} \quad \Longrightarrow\left\{t^{\bullet} \tilde{N} z^{\bullet}: t \leq z \in C\right\}
$$

holds in $\tilde{\mathbf{W}}_{\mathbf{A}}^{p}$ for every interpretation •. As in the previous proof, we may assume that $\left(t^{\bullet}, z^{\bullet}\right)$ is stable for every conclusion $t \leq z$ in $C$. Thus, $(x, z) \in$ $D C(q)$ implies that $\left(x^{\bullet}, z^{\bullet}\right)$ is stable by $(\$)$. But this time stability may not hold for some premises.

So let $t \leq z$ be a premise such that $\left(t^{\bullet}, z^{\bullet}\right)$ is not stable. Then by $(\$)$, we can identify the set $\left\{x_{1}, \ldots, x_{n}\right\}$ of variables in $t$ such that $\left(x_{i}^{\bullet}, z^{\bullet}\right)$ is not stable for any $1 \leq i \leq n$ (thus, $x_{i}^{\bullet} \notin A^{*}, z^{\bullet} \notin A^{\circ}$ and $\left.\left(x_{i}, z\right) \notin D C(q)\right)$. Let us write $t=t\left(x_{1}, \ldots, x_{n}\right)$. By Lemma 5.1(2) and (3), we have

$$
g \leq \bar{z}^{\bullet}, \quad \bar{x}_{i}^{\bullet} \leq h \quad(\text { for } 1 \leq i \leq n) .
$$

By the definition of a semi-anchored clause, two cases can occur.

(1) First, assume that there is a premise $t_{s} \leq z$ with $t_{s}=t\left(y_{1}, \ldots, y_{n}\right)$ and $\left(y_{1}, z\right), \ldots,\left(y_{n}, z\right) \in D C(q)$. Then $\left(t_{s}^{\bullet}, z^{\bullet}\right)$ is stable by $(\$)$. Hence, by Lemma 5.1(1), we have $\bar{t}_{s}^{\bullet} \leq \bar{z}^{\bullet}$. Since $(g, h)$ is a gap, we have that either $\bar{y}_{i}^{\bullet} \leq g$ or $h \leq \bar{y}_{i}^{\bullet}$ for each $1 \leq i \leq n$. We distinguish two cases.

(a) There is some $y_{i}^{\bullet}$ such that $\bar{y}_{i}^{\bullet} \leq g$. As $\left(y_{i}, z\right) \in D C(q)$, there is a conclusion $u \leq z_{i}$ in $C$ such that $u$ contains $y_{i}$. Hence, we have $\bar{u}^{\bullet} \leq \bar{y}_{i}^{\bullet} \leq g \leq \bar{z}^{\bullet}$ by integrality and (!). So we have obtained a true conclusion $u^{\bullet} \tilde{N} z^{\bullet}$.

(b) For every $y_{i}^{\bullet}$, we have $h \leq \bar{y}_{i}^{\bullet}$. We then have $\bar{x}_{i}^{\bullet} \leq h \leq \bar{y}_{i}^{\bullet}$ by (!). Hence, we obtain $\bar{t}^{\bullet}={\overline{t\left(x_{1}, \ldots, x_{n}\right)}}^{\bullet} \leq{\overline{t\left(y_{1}, \ldots, y_{j}\right)}}^{\bullet}=\bar{t}_{s}^{\bullet} \leq z^{\bullet}$. In other words, we have that for the unstable premise $t^{\bullet} \tilde{N} z^{\bullet}$, the inequation $\bar{t}^{\bullet} \leq \bar{z}^{\bullet}$ holds in $\mathbf{A}$.

(2) Assume now that there is a premise $t \leq w$ with $\left(x_{i}, w\right) \in D C(q)$ for some $1 \leq i \leq n$. Since $x_{i}^{\bullet} \notin A^{*}$, this means $w^{\bullet} \in A^{\circ}$, so $\left(t^{\bullet}, w^{\bullet}\right)$ is stable. Hence, by Lemma $5.1(1)$, we have $\bar{t}^{\bullet} \leq \bar{w}^{\bullet}$. As $(g, h)$ is a gap, we have that either $\bar{w}^{\bullet} \leq g$ or $h \leq \bar{w}^{\bullet}$.

(a) In case $h \leq \bar{w}^{\bullet}$, note that there is a conclusion $u \leq w$ such that $u$ contains $x_{i}$. By integrality and Lemma 5.1(2), we have $\bar{u}^{\bullet} \leq \bar{x}_{i}^{\bullet} \leq$ $h \leq \bar{w}^{\bullet}$, which means that we have a true conclusion $u^{\bullet} \tilde{N} w^{\bullet}$. 
(b) In case $\bar{w}^{\bullet} \leq g$, we have $\bar{t}^{\bullet} \leq \bar{w}^{\bullet} \leq g \leq \bar{z}^{\bullet}$ by (!). In other words, we have that for the unstable premise $t^{\bullet} \tilde{N} z^{\bullet}$, the inequation $\bar{t}^{\bullet} \leq \bar{z}^{\bullet}$ holds in $\mathbf{A}$.

Summing up, for any unstable premise $t \tilde{N} z$, we have shown that either a conclusion is true (cases (1a) and (2a)), in which case we are done, or that $\bar{t}^{\bullet} \leq \bar{z}^{\bullet}$ holds in $\mathbf{A}$ (cases (1b) and (2b)). Recall that the latter inequation is also true for all the stable premises by Lemma 5.1(1). Hence, our claim follows just by applying $\left\{\bar{t}^{\bullet} \leq_{\mathbf{A}} \bar{z}^{\bullet}: t \leq z \in P\right\} \quad \Longrightarrow\left\{\bar{t}^{\bullet} \leq_{\mathbf{A}} \bar{z}^{\bullet}: t \leq z \in C\right\}$, which holds since $\mathbf{A}$ satisfies $(q)$.

To state our main theorem, let us call an equation semi-anchored if it is equivalent to a set of semi-anchored analytic clauses in the integral FL chains.

Theorem 8.7. Let $\mathrm{V}$ be a nontrivial subvariety of $\mathrm{FL}_{\mathrm{i}}^{\ell}$ defined by a set of semi-anchored equations. Then $\mathrm{V}$ is densifiable, so is standard complete.

Proof. Let $Q$ be the set of semi-anchored analytic clauses equivalent to the defining equations of $\mathbf{V}$. Let $\mathbf{A} \in \mathbf{V}$ be a chain with a gap $(g, h)$. Then $\mathbf{A}$ satisfies all the clauses in $Q$, so by the previous lemma, $\tilde{\mathbf{W}}_{\mathbf{A}}^{p}$ satisfies $\left(q^{N}\right)$ for all $(q) \in Q$. Hence, $\tilde{\mathbf{W}}_{\mathbf{A}}^{p+}$, filling the gap $(g, h)$ of $\mathbf{A}$, satisfies $Q$ by Theorem 7.6 , i.e., $\tilde{\mathbf{W}}_{\mathbf{A}}^{p+} \in \mathrm{V}$.

\section{Densification of subvarieties of $\mathrm{FL}_{\mathrm{e}}^{\ell}$}

Now we turn our attention to subvarieties of $\mathrm{FL}_{e}^{\ell}$ algebras. The situation here is considerably more complicated than for $\mathrm{FL}_{\mathrm{i}}^{\ell}$, and we have no idea how to deal with $\mathcal{P}_{3}$, or even $\mathcal{N}_{2}$, equations uniformly. We thus limit ourselves to the subvarieties of $\mathrm{FL}_{\mathrm{e}}^{\ell}$ defined by knotted axioms $x^{m} \leq x^{n}$, with distinct $m, n>1$, translating in our framework the results in [2]. To begin with, any knotted axiom implies a curious fact, that $y x^{k} \leq 1$ holds if and only if $y x^{l} \leq 1$, as long as $k, l \geq 1$.

Lemma 9.1. Let $\mathbf{A}$ be a commutative $F L$ chain satisfying $x^{m} \leq x^{n}$ for some distinct $m, n>0$. Then $\mathbf{A}$ satisfies the following quasiequations.

$$
\begin{aligned}
& x x y \leq 1 \quad \Longrightarrow \quad x y \leq 1 \text {, } \\
& x y \leq 1 \quad \Longrightarrow \quad x x y \leq 1 \text {. }
\end{aligned}
$$

Proof. Notice that $\left(c_{1}\right)$ and $\left(w_{1}\right)$ are mutually derivable in commutative FL chains. Therefore, we will only show that $\left(c_{1}\right)$ holds in case $m<n$. In case $n<m$, one can prove in a symmetric way that $\left(w_{1}\right)$ holds. Given $a, b \in A$, assume that $a a b \leq 1$ holds in $\mathbf{A}$. This implies (1): $a^{2 n} b^{n} \leq 1$. We have either $1 \leq a$ or $a \leq 1$. In the former case, we immediately obtain $a b \leq a a b \leq 1$. In the latter case, we have (2): $a^{n} \leq a^{l}$ for every $l \leq n$. Now choose $k, l \in \mathbb{N}$ such that $2 n=k(n-m)+l$ and $m \leq l<n$. Notice that since $m \leq l$, we have by the knotted axiom, $a^{l}=a^{(l-m)} a^{m} \leq a^{(l-m)} a^{n}=a^{l} a^{n-m}$. Hence, we get (3): 
$a^{l} \leq a^{l} a^{k(n-m)}=a^{2 n}$. By (1)-(3), we obtain $a^{n} b^{n} \leq a^{l} b^{n} \leq a^{2 n} b^{n} \leq 1$. Since $\mathbf{A}$ is a chain, one can easily show that $a b \leq 1$ follows from the latter.

Another important fact is that any knotted axiom is equivalent to a simple analytic quasiequation in commutative FL chains.

Lemma 9.2. Let $\mathbf{A}$ be a commutative $F L$ chain. Then $\mathbf{A}$ satisfies $x^{m} \leq x^{n}$, for distinct $m, n>0$, if and only if it satisfies the following quasiequation:

$$
x_{1}^{n} \leq z \text { and } \cdots \text { and } x_{m}^{n} \leq z \Longrightarrow x_{1} \cdots x_{m} \leq z . \quad\left(\text { knot }_{m}^{n}\right)
$$

Proof. Assume $\left(\right.$ knot $\left._{m}^{n}\right)$ holds in A. Given $a \in A$, we interpret all $x_{1}, \ldots, x_{m}$ by $a$ and $z$ by $a^{n}$. Then all the premises hold, and the conclusion is $a^{m} \leq a^{n}$.

Conversely, assume $x^{m} \leq x^{n}$ holds in A. Suppose that $a_{1}^{n} \leq b, \ldots, a_{m}^{n} \leq b$ hold for $a_{1}, \ldots, a_{m}, b \in A$. Our goal is to show $a_{1} \cdots a_{m} \leq b$. Since $\mathbf{A}$ is a chain, there is a maximum among $a_{1}, \ldots, a_{m}$, say $a_{k}$. Then we have $a_{1} \cdots a_{m} \leq a_{k}^{m} \leq a_{k}^{n} \leq b$.

Notice that since the knotted axioms are in the class $\mathcal{N}_{2}$, we could have just used the procedure in $[8,9]$ to obtain equivalent analytic quasiequations. The quasiequations we have considered here are a simplified version, which are equivalent to the axioms only when we restrict to chains.

Lemma 9.3. Let $\mathbf{A}$ be a commutative $F L$ chain with a gap $(g, h)$, satisfying $\left(\right.$ knot $\left._{m}^{n}\right)$ for some distinct $m, n>1$. The residuated frame $\tilde{\mathbf{W}}_{\mathbf{A}}^{p}$ defined in Section 6 satisfies $\left(\right.$ knot $\left._{m}^{n N}\right)$.

Proof. We need to show that $\tilde{\mathbf{W}}_{\mathbf{A}}^{p}$ satisfies

$$
x_{1}^{n} \tilde{N} z \text { and } \cdots \text { and } x_{m}^{n} \tilde{N} z \Longrightarrow x_{1} \cdots x_{m} \tilde{N} z, \quad\left(k_{n o t}^{n N}\right)
$$

for every $x_{1}, \ldots, x_{m} \in W=A \times \mathbb{N}$ and $z \in \tilde{W}^{\prime}=W \times W^{\prime}=W \times(A \cup\{p\})$. The conclusion is stable if and only if all the premises are. If this is the case, the claim easily follows from Lemma 6.1 and from the fact that $\mathbf{A}$ satisfies $\left(\right.$ knot $\left._{m}^{n}\right)$.

So assume that some of the premises violate stability, for instance, without loss of generality, $x_{1}^{n} \tilde{N} z, \ldots, x_{k}^{n} \tilde{N} z$ with $1 \leq k \leq m$. This means that there are $a_{1}, \ldots, a_{m}, b \in A$ and natural numbers $e_{1}, \ldots, e_{k} \geq 1$ such that

$$
z=(b, p), \quad x_{i}=a_{i} p^{e_{i}}(1 \leq i \leq k), \quad x_{j}=a_{j}(k+1 \leq j \leq m) .
$$

Then $\left(\right.$ knot $\left._{m}^{n N}\right)$ amounts to:

$$
\left.\begin{array}{ccc}
a_{1}^{n} b h^{n e_{1}-1} \leq 1, & \ldots, & a_{k}^{n} b h^{n e_{k}-1} \leq 1, \\
a_{k+1}^{n} b \leq g, & \ldots, & a_{m}^{n} b \leq g
\end{array}\right\} \quad \Longrightarrow \quad a_{1} \cdots a_{m} b h^{e_{1}+\cdots+e_{k}-1} \leq 1 .
$$

By combining all the premises on the first line and by Lemma 9.1 (noting that $n>1$ ), we obtain (*): $a_{1}^{n} \cdots a_{k}^{n} b^{k} h^{l} \leq 1$, for any $l \geq 1$. By combining all those on the second line, we obtain (**): $a_{k+1}^{n} \cdots a_{m}^{n} b^{m-k} \leq g^{m-k} \leq h^{m-k}$.

If $e_{1}+\cdots+e_{k}-1 \geq 1$, the two inequalities $(*)$ and $(* *)$ with $l:=m-k+1$ imply $a_{1}^{n} \cdots a_{m}^{n} b^{m} h \leq 1$, which leads to the conclusion by Lemma 9.1 . 
Otherwise $k=1$ and $e_{1}=1$. Since $m>1$, we have $m-k \geq 1$. Hence, $(*)$ and $(* *)$ with $l:=m-k$ implies $a_{1}^{n} \cdots a_{m}^{n} b^{m} \leq 1$, which leads to the conclusion.

Finally, we obtain the main theorem of this section.

Theorem 9.4. Let $\mathrm{V}$ be the subvariety of $\mathrm{FL}_{\mathrm{e}}^{\ell}$ defined by $x^{m} \leq x^{n}$ with distinct $m, n>1$. Then $\mathrm{V}$ is densifiable, and so is standard complete.

Proof. Let $\mathbf{A} \in \mathrm{V}$ be a chain with a gap $(g, h)$. By Lemmas 9.2 and $9.3, \tilde{\mathbf{W}}_{\mathbf{A}}^{p}$ satisfies $\left(k n o t_{m}^{n N}\right)$. Hence, $\tilde{\mathbf{W}}_{\mathbf{A}}^{p+}$, filling the gap $(g, h)$ of $\mathbf{A}$, satisfies $\left(k n o t_{m}^{n}\right)$ by Theorem 7.6, i.e., $\tilde{\mathbf{W}}_{\mathbf{A}}^{p+} \in \mathrm{V}$.

Notice that $x^{m} \leq x^{0}$ with $m>0$ is equivalent to the integrality $x \leq 1$, that has already been dealt with; indeed, the former implies $x \leq(1 \vee x)^{m} \leq$ $(1 \vee x)^{0}=1$. Likewise, $x^{0} \leq x^{n}$ with $n>0$ is equivalent to $1 \leq x$, which defines the trivial variety.

The only remaining cases are $x^{m} \leq x^{1}$ and $x^{1} \leq x^{n}$ with $m, n>1$, which are respectively equivalent to $x^{2} \leq x$ and $x \leq x^{2}$ in $\mathrm{FL}^{\ell}$. Unfortunately, our result in this section, as well as its proof theoretic origin [2], does not cover these cases. [26] shows proof-theoretically only that the subvariety of $\mathrm{FL}^{\ell} \mathrm{ax}$ iomatized by both $x^{2} \leq x$ and $x \leq x^{2}$ is densifiable. In a recently submitted work [3], all these cases are addressed by general proof-theoretic means. Translating them into our algebraic framework would require the construction of a residuated frame different from the one given in Section 6 .

\section{Final remarks and open problems}

The results presented here subsume most of the results on strong standard completeness of fuzzy logics in the literature $[4,6,14,15,16,21,23,24,26,30]$.

Unfortunately, $\mathcal{N}_{3}$-subvarieties (i.e., subvarieties defined by $\mathcal{N}_{3}$ equations) of $\mathrm{FL}^{\ell}$, or even $\mathrm{FL}_{\text {eio }}^{\ell}$, cannot be dealt with by our method. This is the case, for instance, for the varieties corresponding to basic logic, Eukasiewicz logic, product logic, WCMTL and IMTL (see, e.g., [13, 14, 15, 28, 20]). It is certainly a limitation of our approach, but notice that these varieties only enjoy the finite strong form of standard completeness $\left(E \models \mathrm{v} s=t \Leftrightarrow E \models \mathrm{V}_{[0,1]} s=t\right.$ for finite $E$ ), and it has actually been proved that none of them admits the strong form studied in this paper.

It is an open problem to what extent the $\mathcal{N}_{2}$-subvarieties of $\mathrm{FL}_{\mathrm{e}}^{\ell}$ admit densification and standard completeness. Section 9 only gives a partial solution (for knotted axioms $x^{m} \leq x^{n}$ with $m, n>1$ ). In this paper, we have not considered involutive subvarieties of FL, i.e., those defined by $-\sim x=\sim-x=$ $x$. For involutive $\mathrm{FL}_{\mathrm{e}}^{\ell}$, which corresponds to involutive monoidal $t$-norm logic, strong standard completeness has been proved algebraically in [15] and prooftheoretically in [26]. We believe that this result can be reproved by employing involutive residuated frames of [18]. On the other hand, an important open 
problem in this direction is the standard completeness of involutive $\mathrm{FL}_{\mathrm{e}}^{\ell}$, which correspond to involutive uninorm logic, for which we are not sure whether our method applies or not.

Acknowledgments. Open access funding provided by TU Wlen(TUW). We thank Agata Ciabattoni, Nikolaos Galatos and Rostislav Horčík for the fruitful discussions and suggestions which strongly influenced this work.

\section{REFERENCES}

[1] Baaz, B., Zach, R.: Hypersequents and the proof theory of intuitionistic fuzzy logic. In: Computer Science Logic (Fischbachau, 2000). Lecture Notes in Comput. Sci., vol. 1862, pp. 187-201. Springer, Berlin (2000)

[2] Baldi, P.: A note on standard completeness for some extensions of uninorm logic. Soft Computing 18, 1463-1470 (2014)

[3] Baldi, P., Ciabattoni, A.: Standard completeness for Uninorm-based logics. In: G. Dueck, editor, IEEE 45th International Symposium on Multiple-Valued Logic, ISMVL 2015, Waterloo, Canada. Proceedings, pp. 78-83, 2015

[4] Baldi, P., Ciabattoni, A., Spendier, L.: Standard completeness for extensions of MTL: an automated approach. In: Logic, Language, Information and Computation. Lecture Notes in Comput. Sci., vol. 7456, pp. 154-167. Springer, Heidelberg (2012)

[5] Banaschewski, B.: Hüllensysteme und Erweiterungen von Quasi-Ordnungen. Z. Math. Logik Grund. Math. 2, 35-46 (1956)

[6] Ciabattoni, A., Esteva, F., Godo, L.: T-norm based logics with $n$-contraction. Neural Network World 12, 441-453 (2002)

[7] Ciabattoni, A., Galatos, N., Terui, K.: From axioms to analytic rules in nonclassical logics. In: Logic in Computer Science, 2008, pp. 229-240. IEEE (2008)

[8] Ciabattoni, A., Galatos, N., Terui, K.: MacNeille completions of FL-algebras. Algebra Universalis 66, 405-420 (2011)

[9] Ciabattoni, A., Galatos, N., Terui, K.: Algebraic proof theory for substructural logics: cut-elimination and completions. Ann. Pure Appl. Logic 163, 266-290 (2012)

[10] Ciabattoni, A., Galatos, N., Terui, K.: Algebraic proof theory: hypersequents and hypercompletions (preprint)

[11] Ciabattoni, A., Metcalfe, G.: Density elimination and rational completeness for first-order logics. In: Logical Foundations of Computer Science. Lecture Notes in Comput. Sci., vol. 4514, pp. 132-146. Springer, Berlin (2007)

[12] Ciabattoni, A., Metcalfe, G.: Density elimination. Theoret. Comput. Sci. 403, 328-346 (2008)

[13] Cignoli, R., Esteva, F., Godo, L., Torrens, A.: Basic fuzzy logic is the logic of continuous t-norms and their residua. Soft Computing 4, 106-112 (2000)

[14] Cintula, P., Esteva, F., Gispert, J., Godo, L., Montagna, F., Noguera, C.: Distinguished algebraic semantics for t-norm based fuzzy logics: methods and algebraic equivalencies. Ann. Pure Appl. Logic 160, 53-81 (2009)

[15] Esteva, F., Gispert, J., Godo, L., Montagna, F.: On the standard and rational completeness of some axiomatic extensions of the monoidal t-norm logic. Studia Logica 71, 199-226 (2002)

[16] Esteva, F., Godo, L.: Monoidal t-norm based logic: towards a logic for left-continuous t-norms. Fuzzy Sets and Systems 124, 271-288 (2001)

[17] Galatos, N., Horčík, R.: Densification via polynomials, languages and frames (preprint)

[18] Galatos, N., Jipsen, P.: Residuated frames with applications to decidability. Trans. Amer. Math. Soc. 365, 1219-1249 (2013)

[19] Galatos, N., Jipsen, P., Kowalski, T., Ono, H.: Residuated Lattices: An Algebraic Glimpse at Substructural Logics. Studies in Logic and the Foundations of Mathematics, vol. 151. Elsevier, Amsterdam (2007) 
[20] Horčík, R.: Standard completeness theorem for ПMTL logic. Arch. Math. Logic 44, 413-424 (2005)

[21] Horčík, R.: Algebraic semantics. In: Cintula, P., Hajek, P., Noguera, C. (eds.) Handbook of Mathematical Fuzzy Logic, pp. 283-353. College Publications (2011)

[22] Horčík, R., Noguera, C., Petrík, M.: On n-contractive fuzzy logics. MLQ Math. Log. Q. 53, 268-288 (2007)

[23] Jenei, S., Montagna, F.: A proof of standard completeness for Esteva and Godo's Logic MTL. Studia Logica 70, 183-192 (2002)

[24] Jenei, S., Montagna, F.: A proof of standard completeness for non-commutative monoidal t-norm logic. New Network World 13, 481-489 (2003)

[25] Metcalfe, G.: Proof theory for mathematical fuzzy logic. In: Cintula, P., Hajek, P., Noguera, C. (eds.) Handbook of Mathematical Fuzzy Logic, pp. 283-353. College Publications (2011)

[26] Metcalfe, G., Montagna, F.: Substructural fuzzy logics. J. Symbolic Logic 7, 834-864 (2007)

[27] Metcalfe, G., Olivetti, N., Gabbay, D.: Proof Theory for Fuzzy Logics. Applied Logic Series, vol. 36. Springer, New York (2009)

[28] Montagna, F., Noguera, C., Horčík, R.: On weakly cancellative fuzzy logics. J. Logic Comput. 16, 423-450 (2006)

[29] Schmidt, J.: Zur Kennzeichnung der Dedekind-MacNeilleschen Hülle einer geordneten Hülle. Arch. Math. (Basel) 7, 241-249 (1956)

[30] Wang, S.M.: Uninorm logic with the $n$-potency axiom. Fuzzy Sets and Systems 205, 116-126 (2012)

[31] Wang, S.M., Zhao, B.: HpsUL is not the logic of pseudo-uninorms and their residua. Log. J. IGPL 17, 413-419 (2009)

\section{PAOLO BALDi}

Department of Computer Languages, Vienna University of Technology, Favoritenstrasse 9-11, 1040 Wien, Austria

e-mail: baldi@logic.at

\section{Kazushige Terui}

Research Institute for Mathematical Sciences, Kyoto University, Kitashirakawa Oiwakecho, Sakyo-ku, Kyoto 606-8502, Japan

e-mail: terui@kurims.kyoto-u.ac.jp

Open Access This article is distributed under the terms of the Creative Commons Attribution 4.0 International License (http://creativecommons.org/licenses/by/4.0/), which permits unrestricted use, distribution, and reproduction in any medium, provided you give appropriate credit to the original author(s) and the source, provide a link to the Creative Commons license, and indicate if changes were made. 